\title{
THE EFFECTS OF A COMBINED SUPPLEMENTATION OF CREATINE AND SODIUM BICARBONATE ON REPEATED SPRINT PERFORMANCE
}

\author{
A Thesis \\ presented to \\ the Faculty of California Polytechnic State University, \\ San Luis Obispo
}

\author{
In partial fulfillment \\ of the requirements for the degree of Master of Science in Kinesiology \\ California Polytechnic State University, San Luis Obispo \\ by \\ James Jeremy Barber
}

September 2010 
(C) 2010

James Jeremy Barber ALL RIGHTS RESERVED 


\section{Committee Membership}

TITLE:

The Effects of a Combined Supplementation of Creatine and Sodium Bicarbonate on Repeated Sprint Performance

AUTHOR: James Jeremy Barber

DATE SUBMITTED: $\quad$ 09/2010

COMMITTEE CHAIR: $\quad$ Todd Hagobian, Ph.D.

COMMITTEE MEMBER: Ann McDermott, Ph.D., M.S., LDN

COMMITTEE MEMBER: Karen McGaughey, Ph.D.

COMMITTEE MEMBER: Jennifer Olmstead, M.S., C.S.C.S. 


\begin{abstract}
The Effects of a Combined Supplementation of Creatine and Sodium Bicarbonate on Repeated Sprint Performance James Jeremy Barber
\end{abstract}

There is well-established research that suggests both creatine and sodium bicarbonate are effective ergogenic aids. However, only one published study has examined the combined effects of creatine and sodium bicarbonate. The primary purpose of this study was to determine if a combined supplementation of creatine monohydrate and sodium bicarbonate would further enhance the well-documented effects of creatine supplementation alone on repeated sprint performance. Thirteen healthy and fit males (Mean age $=21.15 \pm 0.65$ years and mean VO2 max $=66.72 \pm 5.78)$ participated in this experimental study using a double-blinded crossover study design in which each subject was used as his own control. All subjects completed 3 conditions, followed by a 3-week washout period between each condition: 1) Placebo (Pl; $5 \mathrm{~g}$ maltodextrin + $0.5 \mathrm{~g} / \mathrm{kg}$ maltodextrin), 2) Creatine (Cr; $5 \mathrm{~g}+0.5 \mathrm{~g} / \mathrm{kg}$ maltodextrin), and 3) Creatine plus sodium bicarbonate $(\mathrm{Cr}+\mathrm{Sb} ; 5 \mathrm{~g}+0.5 \mathrm{~g} / \mathrm{kg}$ sodium bicarbonate). Each condition was a 2-day supplementation. In the morning after each supplementation, peak power, RPP, mean power, RMP, fatigue index, and perceptions of fatigue and GI distress were assessed during six 10second repeated Wingate tests. Blood bicarbonate, $\mathrm{pH}$, and lactate were measured 5 minutes before testing and immediately after the last Wingate sprint.

The main findings were; 1) $\mathrm{Cr}+\mathrm{Sb}$ produced $7 \%$ greater relative peak power and $4.6 \%$ greater peak power values than placebo, and 2) $\mathrm{Cr}+\mathrm{Sb}$ demonstrated the greatest attenuation of decline in relative peak power over six repeated sprints. However, in contrast to our hypotheses, 
no benefits from either supplementation were observed for relative mean power, fatigue index, and perception of fatigue. Considering that this current study found benefits from combining creatine and sodium bicarbonate, it suggests that combining the supplements may improve repeated sprint performance. Future research on a greater sample size, a specific athletic population, various exercise modes, and comparing results with a sodium bicarbonate alone supplementation would be beneficial in determining if this combined supplementation is worthwhile. 


\section{Acknowledgements}

First and foremost, I would like to express my deepest appreciation to my committee chair, Dr. Todd Hagobian. Dr. Hagobian never hesitated to answer my questions, review drafts, and give suggestions throughout the entire process. I am very appreciative of Dr. Hagobian's trust in my abilities perform all experiments and procedures. Without his encouragement, knowledge, and patience, this thesis would not have been possible.

I would also like to extend my appreciation to Dr. Ann McDermott, and Jennifer Olmstead, my thesis committee members, for their time, suggestions on research protocol, and enthusiasm for this thesis.

I am especially appreciative of Dr. Karen McGaughey, committee member, for her numerous efforts in helping with the statistics for this thesis. Not only did she spend hours helping me analyze the statistics of this thesis, she taught me the nuts and bolts of statistics.

I would also like to extend my gratitude to my graduate advisor, Dr. Robert Clark. Dr. Clark never hesitated to give valuable insight into the methods of this study. I thank Dr. Clark for his expertise and help with the use of the Velotron Cycler Ergometer.

I would like to further extend my appreciation to Dr. Derek Marks, my undergraduate mentor. Dr. Marks initially helped formulate the idea and theory behind this thesis. Without his inspiration and constant motivation, this thesis would not be possible.

I also want to give thanks to my good friends Chris Borgard, Jamie Bennett, David Norris, James Ramirez, and Arya Alami for their support and assistance in data collection. Finally, I extend a special thank you to my parents, Mike and Lilia Barber, brother Jon, and Melaney, for supporting me through all my endeavors. Your unwavering belief in me has given me the strength to accomplish anything I set forth to achieve. 
List of Tables

\section{Chapter 1}

Introduction

ix

Background

Purpose

Hypotheses

Significance of Study

Definition of Terms

Chapter 2

Review of Literature

Review of Creatine Studies

Review of Creatine Dosing

Review of Sodium Bicarbonate Studies

Review of Sodium Bicarbonate Dosing

Review of Combining Creatine and Sodium Bicarbonate

Chapter 3

Methods and Procedures

Participants

Preliminary Tests

Supplementation Procedures

Repeated Wingate Test Procedures

Blood Analysis

Statistical Analysis

Power and Sample Size

Pilot Study Results

Limitations

\section{Chapter 4}

Results

Performance Measures

Blood Analysis

36

Perception of Fatigue and GI Distress

45

Chapter 5

Discussion

Conclusion

Recommendations

Appendices

$\begin{array}{ll}\text { A. Informed Consent } & 63\end{array}$

$\begin{array}{ll}\text { B. Screening Questionnaire } & 67\end{array}$

$\begin{array}{ll}\text { C. Fatigue and GI distress questionnaire } & 70\end{array}$

D. Supplementation Recall Sheet $\quad 71$ 
LIST OF TABLES

Table 1: Subject Characteristics

Table 2: Supplementation Model

Table 3: Expected Results

Table 4: Performance Results

Table 5: Perceptions of Fatigue and GI distress 
Figure 1: Power and Sample Size Calculation

Figure 2: Pilot Data: Repeated Wingate Results for sample subject \#1 32

Figure 3: Pilot Data: Repeated Wingate Results for sample subject \#2 33

Figure 4.1: Main Effect Chart for Peak Power Output $\quad 37$

Figure 4.2: Peak Power Output 37

Figure 5.1: Main Effect Chart for Relative Peak Power Output 39

Figure 5.2: Relative Peak Power Output 39

Figure 6.1: Main Effect Chart for Mean Power Output $\quad 40$

Figure 6.2: Mean Power Output $\quad 41$

Figure 7: Relative Mean Power Output $\quad 42$

Figure 8: Fatigue Index $\quad 43$

Figure 9: Blood Bicarbonate $\quad 45$

Figure 10: Blood pH $\quad 46$

Figure 11: Blood Lactate $\quad 47$ 


\section{Chapter 1}

\section{Introduction}

\section{Statement of the Problem}

Participation in high school, amateur, and professional athletics has reached an all time high. As more people are participating in athletics than they did two decades ago, athletes are constantly looking for a competitive advantage to further improve their performance (36). This includes, but is not limited to, altering energy or carbohydrate intake, environmental training (e.g., live-high-train low, heat training), and using ergogenic aids. These methods have shown to improve performance in various exercise settings, however, using ergogenic aids has shown to be the most common method to improve performance. Approximately $60 \%$ of all athletes worldwide use ergogenic aids (46).

Although many athletes use ergogenic aids, current research only supports a select few that actually improve performance. The primary purpose of ergogenic aids is to reduce fatigue and increase power output. With high-intensity exercise, potential contributors to fatigue can be related to muscle energy production, a decline in muscle adenosine triphosphate (ATP), or impaired electrochemical events of muscle contraction/relaxation production (44). These decrements in performance can also be related to the accumulation of metabolites during exercise such as hydrogen ions resulting from increased lactic acid formation in high intensity exercise (44). Creatine monohydrate, and sodium bicarbonate, are two widely-studied ergogenic aids that have shown to attenuate the decline in fatigue while also increasing power output in high intensity exercise bouts such as sprinting $(1,3,4,7,8,11,12,16,31,32,55,65,68)$.

Creatine $(\mathrm{Cr})$ may be considered the most widely studied ergogenic aid in the past decade (36). Since its introduction in 1992, creatine has become the most popular and profitable 
ergogenic aid on the market with more than $\$ 300$ million in 2000 sales just in the United States alone (63). Many studies have shown that creatine supplementation increases performance during high-intensity, intermittent exercise (1, 3, 4, 11, 12, 31, 47, 55, 68). Dawson et al. (1995) found that creatine supplementation of $20 \mathrm{~g}$ for 5 days increased peak power $(+4.5 \%)$ and total work performed $(+4.6 \%)$ in subjects performing six repeated cycle sprints. This same study also demonstrated that creatine supplementation provided a lower decline in fatigue over the period of the six repeated sprints. Balsom et al. (1993) found that creatine supplementation increased total work output in ten repeated cycling bouts. Similarly, Ziegenfuss et al. (2002) found that the same $20 \mathrm{~g}$ dose for three days increased power output in six 10-second repeated Wingate sprints. These data suggest that creatine supplementation improves performance during high intensity intermittent exercise.

Creatine's ergogenic benefits stem from its importance in high intensity energy metabolism. Total creatine in skeletal muscle is considered the sum of free creatine (FC) and phosphocreatine (PCr) (stored form), both of which are important in the production of adenosine triphosphate (ATP) during anaerobic activity (54). Of the total creatine stored within skeletal muscle, approximately $40 \%$ is free creatine and $60 \%$ in the form of $\mathrm{PCr}(14)$. Energy is provided to the body from the hydrolysis of ATP into adenosine diphosphate (ADP) and an inorganic phosphate (Pi). During high intensity exercise, creatine acts as a substrate for the formation of ATP by rephosphorylating adenosine diphosphate ADP. When phophorylated by the enzyme creatine kinase $(\mathrm{CK})$, the resulting $\mathrm{PCr}$ can donate its inorganic phosphate required to rephosphorylate ADP (45).

When the PCr stores become depleted in the muscle during maximal intensity exercise, the ability to sustain optimal high-intensity performance decreases (45). Creatine 
supplementation has the ability to increase skeletal muscle stores of creatine and PCr, which may therefore increase the skeletal muscle's ability to increase ATP resynthesis from ADP. Previous studies suggest that creatine supplementation of approximately 20-30 grams for several days significantly raises total muscle creatine content by an additional $25 \%(25,26,28)$. Because the availability of PCr stores in muscle significantly influence the amount of energy generated during brief high intensity exercise, it has been suggested that increasing muscle creatine concentrations may increase the availability of PCr, thus allowing for enhanced ATP resynthesis during exercise and rest intervals of repeated high intensity exercise $(2,12,25)$. Taken together, these studies suggest that supplementing with creatine for several days may enhance intermittent sprint performance such as overall peak power output and maintaining maximal effort slightly longer before peak power is compromised due to fatigue.

In addition to creatine supplementation, several studies have investigated the use of sodium bicarbonate as an ergogenic aid. Current research has shown that ingesting sodium bicarbonate may enhance aspects of sprint performance such as power output, total anaerobic work, and delaying fatigue (7, 8, 32, 38, 39, 40, 41, 42, 65). Lavender and Bird (1989), and Bishop et al. (2004-2005), concluded that sodium bicarbonate supplementation improved power output levels in repeated sprints. These studies suggest that the improvements in performance may be attributed to the enhanced buffering capacity of hydrogen ions resulting from sodium bicarbonate ingestion $(7,8,32)$. During high-intensity exercise, accumulation of hydrogen ions, due primarily to increases in lactic acid within the muscle, lowers muscle $\mathrm{pH}$ (45). This fall in $\mathrm{pH}$ is considered to inhibit muscular contraction in a number of ways. These include, limiting the rate of resynthesis of ATP by inhibiting glycolytic enzymes, inhibiting the release of calcium ions needed for muscular contraction, and impairing the interaction between actin and myosin in 
the muscle cell $(32,51)$.

This process stimulates extracellular buffering mechanisms in which bicarbonate is one of the most important buffering agents. By several mechanisms, bicarbonate has the ability to remove accumulated hydrogen ion $(\mathrm{H}+)$ out of the muscle cell. This reduces the inhibition of muscular contraction and therefore sustains high intensity exercise. Similar to creatine bicarbonate stores are limited. Previous research has shown that ingesting sodium bicarbonate increases bicarbonate concentrations and blood $\mathrm{pH}$ levels $(7$ - 9, 16, 34, 37 - 41, 43 - 45, 51, 53, 65). Taken together these studies suggest that by increasing extracellular buffering agents such as bicarbonate, may improve sprint performance by increasing the ability to remove resulting metabolites such as hydrogen ions and lactate out of the muscle cell $(7,8)$.

Although many studies have addressed the effect of creatine monohydrate and sodium bicarbonate alone on performance, only one published study has examined the combined effect of these supplements even though it is important from a basic science perspective (e.g. mechanism of action of creatine and sodium bicarbonate), as well as practical application (e.g. improving performance in athletics) (45). Mero et al. (2004) found that combining creatine and sodium bicarbonate improved 100-meter swim performance. However, this study was confounded by not having a creatine or sodium bicarbonate alone condition. Therefore, there is no way to quantify how much, if at all, the added effect of the combined supplementation of creatine and sodium bicarbonate actually had on swimming performance. Also, this study was conducted on "national" competitive swimmers, which is not applicable to all athletes. Finally, the supplementation procedures did not technically "combine" the supplements in one drink. Subjects supplemented on creatine for 6 days and, on the $7^{\text {th }}$ day, ceased creatine supplementation and ingested the full dose of sodium bicarbonate. Thus, there is no way of 
determining if the pre-race bicarbonate supplementation overshadowed the effects of the weeklong dose of creatine or vice versa.

The initial study by Mero et al. (2004) provides preliminary data that combining creatine with sodium bicarbonate may further enhance performance compared to either supplement alone. Because creatine and sodium bicarbonate function in different metabolic pathways and have no known interactive effects, combining creatine with sodium bicarbonate may have greater performance benefits than that of creatine or bicarbonate alone.

\section{Statement of Purpose}

The primary purpose of this study was to determine whether a combined supplementation of creatine monohydrate and sodium bicarbonate would further enhance the well-documented effects of creatine supplementation alone on repeated sprint performance.

\section{Research Hypotheses}

It was hypothesized that:

1. Supplementation of creatine monohydrate combined with sodium bicarbonate will significantly increase peak power, relative peak power (RPP), mean power, and relative mean power (RMP) compared to creatine alone and placebo.

2. Supplementation of creatine monohydrate combined with sodium bicarbonate will significantly lower fatigue index compared to creatine alone and placebo.

3. Supplementation of creatine monohydrate combined with sodium bicarbonate will significantly lower perceived feelings of fatigue compared to creatine alone and placebo.

4. Supplementation of creatine monohydrate combined with sodium bicarbonate will significantly increase blood bicarbonate, lactate, and $\mathrm{pH}$ levels immediately pre and post exercise compared to creatine alone and placebo. 


\section{Significance}

To our knowledge, this is the first study to investigate whether a combined supplementation of creatine monohydrate and sodium bicarbonate will further enhance the welldocumented effects of creatine supplementation alone on repeated sprint performance. This study will give further insight into the combined effects of these two supplements on performance. Many coaches, at all levels, advise the use of creatine and/or sodium bicarbonate to maximize the training and performance of athletes. These supplements are also easily accessible. Creatine is a readily available over-the-counter supplement while sodium bicarbonate can be found in any supermarket for under a dollar. The results from this study can give insight to coaches as well as athletes, to which supplementation, will be most effective in sports that involve repeated sprints.

\section{Definition of Terms}

The following terms and abbreviations are defined as used in the study.

ATP (Adenosine triphosphate): A high-energy phosphate compound from which the body derives its energy.

$\underline{\mathrm{Cr}}$ (Creatine Monohydrate): Substance found in skeletal muscles mainly in the form of phosphocreatine. Supplementing on creatine is theorized to increase $\mathrm{PCr}$ stores better maintaining muscle ATP levels.

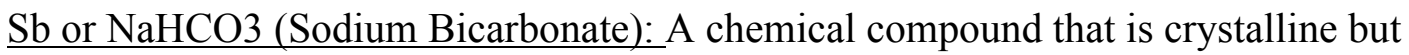
often appears as a fine powder. Supplementing on sodium bicarbonate is theorized to aid in the acid-base balance system by buffering hydrogen ions.

Wingate: Cycle ergometer test measure anaerobic peak powers, mean power, total work, and fatigue index.

Relative Peak Power: A measure of anaerobic power relative to a person's body weight in kilograms. It is expressed in watts/kilogram.

Fatigue Index: A measure of the rate of fatigue over a specific time period. It is expressed in watts/time. 


\section{Chapter 2}

\section{$\underline{\text { Review of Literature }}$}

\section{Introduction}

Creatine monohydrate and sodium bicarbonate are two widely studied ergogenic aids on high intensity performance. With extremely high rates of energy turnover during high intensity exercise, muscle performance rapidly decreases (3). This decrease in performance has been attributed to both homeostatic changes within the muscle and the depletion of energy substrates (3). Ingesting creatine and sodium bicarbonate, however, is thought to counter this rapid decline in performance by replenishing substrates and buffering inhibiting metabolites during exercise.

There is well-documented research on each of these ergogenic aids separately, however, only one study has examined the effectiveness of combining the two supplements on exercise performance (45). The primary purpose of this study is determine if a combined supplementation of creatine monohydrate and sodium bicarbonate will further enhance the well documented effects of creatine supplementation alone on repeated sprint performance. This literature review will evaluate the current research of creatine and sodium bicarbonate's ergogenic effect along with their combination.

\section{Creatine Supplementation}

Creatine's ability to enhance rephosphorylation of ADP has brought an abundance of research on its ergogenic effect on various exercise modes and durations. The most beneficial effects of creatine have been seen during short, high intensity exercise bouts as well as repeated sprint bouts $(1,3,4,11,12,31,47,55,68)$.

Early research by Greenhaff et al. (1993) found that while supplementing on creatine, subjects significantly increased muscle peak torque production in comparison to a placebo-fed 
control group. In that study, 12 subjects undertook 5 bouts of 30 maximal voluntary isokinetic contractions with 1-minute rest periods. It was concluded that the increases in creatine concentration provided more efficient ATP resynthesis during exercise (25). Birch et al. (1994) examined creatine supplementation on performance during $3 \times 30$-second bouts of maximal isokinetic cycling. In that study, creatine ingestion significantly increased peak power output in bout 1 and increased total work completed in bouts 1 and 2. Furthermore, McNaughton et al. (1998) found similar positive effects on performance while examining creatine's effect on kayak ergometry for 90-300 seconds. The results of this study indicate that creatine supplementation can significantly increase the amount of work accomplished during kayak ergometer performance at durations ranging from 90 to 300 seconds (42).

There has been much research conducted on creatine's effectiveness in repeated sprints. Given that the importance of $\mathrm{PCr}$ in energy production in these sprint settings increases as the duration of the sprint decreases, the ergogenic effect of creatine may be more important during shorter (40-100 meters) sprints than longer sprints (61). Furthermore, stimulation of muscle $\mathrm{PCr}$ from increased resynthesis may contribute to enhanced recovery during the rest period of repeated or intermittent sprints.

Balsom et al. (1995) studied the ergogenic effect of creatine supplementation on repeated sprints on a stationary cycle ergometer. Five 6-second sprints were performed with a 30 -second rest period. The final 6-second sprint was followed by a 40 -second rest interval before the completion of a final 10-second sprint. In the 10-second exercise period, subjects maintained a significantly higher power output following creatine supplementation compared to placebo.

Similarly, Ziegenfuss et al. (2002) found that a creatine supplementation improved peak power in sprints 2-6 during a repeated sprint cycle test in college athletes. This protocol 
consisted of 6 ten-second repeated Wingate tests which involves maximal effort cycling at a resistance of $0.75 \mathrm{~kg} / \mathrm{kg}$ bodyweight. Aaserud et al. (1998) found that creatine supplementation delayed the onset of fatigue during repeated running sprints. The exercise test consisted of eight 40-meter maximal sprint runs with a 25 second rest between each sprint. The improvements in sprint performance and delay of fatigue may to be attributed to the increased muscle and blood creatine concentration allowing for more efficient ATP rephosphorylation (1).

More intriguing studies by Dawson et al. (1995) and Skare et al. (2001) involved the examination of creatine loading on single and repeated sprint bouts. Dawson et al. (1995) using a pre-ingestion and post-ingestion design, found that supplementing on creatine monohydrate enhanced repeated short ( 6 x 6 seconds) cycle sprints, however not in the single 10-second bout. In the repeated sprint tests, the creatine-supplemented group improved their total work and peak power scores by $4.5 \%$ and $4.6 \%$ (12). This study had an interesting finding in that the creatine and placebo supplementation had no differences in the single 10-second sprint. This may be attributed to the lack of muscle ADP rephosphorylation in a single sprint bout of 10 seconds (12). In repeated sprints, however, the ability for PCr to shuttle phosphates seems to be enhanced because of the recovery period in between sprint bouts.

Skare et al. (2001) examined the effect of creatine supplementation on repeated 60-meter sprints and maximal 100-meter sprints. Similar to Dawson et al. (1995) findings, the researchers found that creatine supplementation enhanced sprint performance. It was concluded that subjects supplementing on creatine showed decreased sprint times observed in 5 out of 6 intermittent 60 m sprints. Unlike Dawson et al. (1995), it was also noted that subjects achieved significantly better results on a single 100-meter sprint trial. These differences could be that Skare et al. (2001) used a different protocol in running sprints as opposed to cycle sprints.. 
Peyrebrune et al. (1998) also examined the effectiveness of creatine supplementation in single and repeated sprint settings in swimming. The subjects performed a single sprint consisting of 50 yards and a repeated sprint set of $8 \times 50$ yards with 1.5 -minute intervals. The this study found similar results with Dawson et al. (1995) in that creatine had no effect on the single sprint trial but did actually see a significant improvement in repeated sprints. Although creatine did not improve 50-yard swim performance, times in the repeated sprint trials were significantly lower when supplementing on creatine. Like most of the studies touched on previously, it was concluded that the main determinant for this performance improvement may have been an increase in muscle creatine content. This may lead to increased $\mathrm{PCr}$ resynthesis during the rest periods, therefore higher muscle phosphocreatine content in the latter sprints of the $8 \times 50$ yard sprint set (47).

These studies confirm that creatine has a positive effect on repeated sprint performance. In repeated sprints, the rate of $\mathrm{PCr}$ resynthesis during the recovery periods may be further accelerated as a result of increased muscle creatine levels following creatine supplementation. This, in theory, enhances aspects of performance such as al peak power output, and decreasing the onset of fatigue

Creatine supplementation has also shown to improve strength in weightlifting and power exercises $(17,58,63)$. It has been shown that the number of repetitions at a specified percentage of single-repetition maximum increases by $20 \%$ to $30 \%$ after a short-term creatine supplementation period $(17,58$,). Stone et al. (1999) found that peak rate of force development for a single vertical jump and power strength was significantly greater with subjects supplementing on creatine monohydrate versus a placebo. Accordingly, Izqueirdo et al. (2002) found that 5 days of creatine supplementation brought about significant improvements in lower 
body maximal repetitive upper and lower body high power exercise bouts in well-trained male handball players. These studies suggest that creatine supplementation aids in strength and weightlifting exercises.

Although there is an abundance of research suggesting that creatine supplementation has a positive effect on performance, several studies have failed to demonstrate a positive effect (2, 18, 19). In high intensity performance settings, Eckerson et al. (2005) failed to find a significant improvement on anaerobic working capacity in males and females after creatine supplementation. Similarly, Ahmun et al. (2005) found that creatine supplementation failed to demonstrate a positive effect in power output and fatigue index in rugby players. Despite any faults in experimental design, it is important to note that not everyone reacts similarly to supplements. Greenhaff et al. (1994) found that, despite the widely accepted acute dosage of 2030 grams for 5 days, approximately $20-30 \%$ of individuals do not respond to creatine supplementation.

Longer, aerobic exercise modes have also failed to show significant improvements in endurance performance from the use of creatine. Englehart et al. (1998) found that creatine supplementation had no effect on endurance measures such as VO2 max during a "triathlon" simulated protocol. However, interval power performance measured during the end of the exercise protocol significantly increased by $18 \%$. From this study, it may be theorized that although the endurance benefits may not apparent in triathletes, anaerobic benefits such as peak power may still yield positive results in the interval "hill" parts of cycling. Taken together, these studies suggest that:

- Creatine increases high intensity performance, most notably, in intermittent or repeated sprints. 
- Creatine has no significant effect on aerobic exercise performance.

- The primary determinants of benefits to performance may be attributed to increases in increased PCr resynthesis allowing for greater ATP regeneration following creatine supplementation.

\section{Creatine: Dosing Regimens}

It must be noted that since there are various exercise modes and durations, many studies have experimented with several different dosage amounts. It is not the intent of this study to determine the "best" dose, however, for the context of this study, the literature will be used to determine an effective and efficient dose. A classical creatine-loading regimen consists of an initial loading phase of 15-25 grams/day for 4-7 days (61). Early research has concluded that ingesting creatine in the amount of 15-25 grams/day for 4-7 days significantly raises total muscle

creatine content up to $25 \%(4,24,26,28)$. Specifically, Greenhaff et al. (1994) demonstrated that ingestion $20 \mathrm{~g} /$ day for 5 days of creatine was necessary to elevate muscle $\mathrm{TCr}$ concentration by $25 \%$ while also improving repeated bouts of maximal voluntary contraction exercise in 5 of 8 subjects. This dose has been considered safe, effective, and more feasible when taken in separate dose throughout the day. In regards to the administration of creatine, Balsom et al. (1994) found that during a single ingestion of $5 \mathrm{~g}$ of creatine monohydrate, creatine levels rose exponentially after approximately 1 hour. Furthermore, Havenetidis et al. (2003) found that blood creatine levels remain elevated for a short period of time with a half-life of 1-1.5 hours (27). It is theorized, that repeating this dose continuously for 3-4 times a day will maintain increased total creatine levels throughout the day.

Although many studies have found that using the traditional 4-7 day "loading" phase can provide a benefit for performance, previous research suggests that this duration may be 
unnecessary. Harris et al. (1992), found that maximal creatine uptake in the muscle cell occurs during the first 2 days of the traditional loading phase (26). Vandenberge et al. (1999) also reported that 2 days of creatine loading of 25 grams significantly elevated $\mathrm{PCr}$ stores by $11 \%$ as well as increasing torque production in maximal knee extension. This same study found three more days of the same supplementation showed no significant increases in torque production (64). The results from these studies may discourage the use of the traditional 5-day loading phase for athletes interested in benefiting from a pre-race or pre-game supplement. The administration of a 2-day supplementation of creatine may be as effective and more convenient for athletes than a weeklong dose. However, it must be noted that many athletes use creatine for different purposes. For one, many weightlifters use creatine as a training supplement for several weeks to see improvements in overall body mass and strength. However, like the focus of this research, many athletes aim to use creatine for a "direct" ergogenic effect in preparation for a competition in less than a week, similar to acute carbohydrate loading.

In summary, this literature review suggests that, without regards to "non-responders" appropriate oral supplementation of at least 15-25 grams/day for 2-5 days with creatine monohydrate can benefit performance during high intensity exercise by means of increasing muscle and blood free creatine and PCr stores therefore accelerating high intensity energy production.

\section{Creatine: Mechanism of Action}

The physiological mechanisms underlying the effects of creatine supplementation are only partly understood (61). It is believed that creatine works as a reservoir of phosphates required for regenerating adenosine triphosphate (ATP), which is the main fuel source in the muscle during initial high intensity exercise (33). Creatine from endogenous and exogenous 
sources is transferred via the blood to skeletal muscle. Normal plasma creatine concentrations range from 50 to $100 \mu \mathrm{mol} / \mathrm{L}$ (26). For a $70 \mathrm{~kg}$ athlete, this amount is approximately $120 \mathrm{~g}$. Of the total creatine content, $95 \%$ is located within the skeletal muscle with $40 \%$ in the form of free creatine and $60 \%$ in $\mathrm{PCr}(5)$. The uptake of creatine into the muscle occurs actively against a concentration gradient. Following its own biosynthesis or dietary intake, a higher level of creatine is in the blood compared with the muscle. Resultantly, blood borne creatine crosses the muscle cell membrane by means of creatine transporter molecules (67).

During intense exercise, ADP is rephosphorylated to ATP primarily by phophocreatine (PCr), which is the stored form of creatine (36). This immediate energy system is referred to as the "Phosphogean system" or the "ATP-PCr system". When phophorylated by the enzyme creatine kinase $(\mathrm{CK})$, the resulting $(\mathrm{PCr})$ can donate the resulting high-energy phosphate required to rephosphorylate ADP (45). This reaction can be simplified by: $\mathrm{PCr}+\mathrm{ADP} \rightarrow \mathrm{C}+\mathrm{ATP}$

High intensity sprinting sports such as football, soccer, track and field events, and weight lifting, use this means of energy transfer, however, the source of PCr seems to be limited. ATP generated by the ATP-PCr system has shown to sustain maximal muscle contraction for approximately 5 - 15 seconds (23). Greenhaff (1995) reported that the rate of PCr utilization peaks after only 1.28 seconds of maximal effort and declines thereafter.

Sustaining performance in high intensity settings beyond a brief period and recovery requires an additional energy source to replenish ATP. If this does not occur, the energy supply from $\mathrm{PCr}$ is depleted and high intensity movement ceases (36). One way to attenuate the rapid depletion of PCr is to ingest creatine in monohydrate form. Previous studies suggest that increasing the creatine content of skeletal muscles through creatine supplementation may increase free creatine levels in the blood and the muscle, therefore, increasing available $\mathrm{PCr}$ 
stores to rephosphorylate ADP to ATP $(1,3,4,11,12,31,47,55,68)$. Previous studies have shown that loading on a dose of 15-25 grams per day for several days may increase total creatine content up to $25 \%(4,24,26,28)$. Ultimately, this would delay the depletion of PCr and ease the decline in ATP production during intense exercise (31).

Increased free creatine stores resulting from creatine supplementation is thought to aid in the shuttling of high-energy phosphates from the site of muscular contraction to the mitochondrial membrane where ATP is produced. It appears that creatine supplementation may hasten phosphocreatine resynthesis during recovery periods of high-intensity intermittent exercise (10). This may be attributed to the enhancing effect that increased free creatine concentration in muscle has on the rate of flux through the creatine kinase reaction at the mitochondrial membrane during rest periods (10). This hypothesis is supported by studies that found increases in muscle total creatine concentration after creatine ingestion is principally in the form of free creatine $(25,26$,).

\section{Sodium Bicarbonate Supplementation}

Many studies have shown that ingesting sodium bicarbonate (baking soda) can significantly improve performance by enhancing buffering capacity through increasing bicarbonate stores $(7,8,9,16,34,37-41,43-45,51,53,65)$. Similar to creatine, most research on sodium bicarbonate supplementation has been examined on high intense exercise bouts through different exercise modes and have found positive results $(7,8,16,32,38-42,65)$.

McNaughton \& Cedaro (1991) examined sodium bicarbonate supplementation on rowing ergometer performance in elite rowers. Results showed that while ingesting sodium bicarbonate, subjects rowed significantly further than when receiving a placebo during maximal effort rowing for 6 minutes. Blood measures also showed that supplementing on sodium bicarbonate 
significantly increased blood $\mathrm{pH}$ and bicarbonate $(\mathrm{HCO} 3)$ levels. It was concluded that the elevation in bicarbonate ions in the blood may have facilitated a greater amount of removal of hydrogen ions out of the muscle cell (40). McNaughton (1992) later found sodium bicarbonate supplementation improved 60 -second cycle performance. Similar to his previous study, it was also found that resting $\mathrm{pH}$ levels were significantly higher in the bicarbonate-supplemented group compared to placebo.

Lindh et al. (2008) found similar benefits of sodium bicarbonate supplementation on swimming performance. The researchers found that 200-meter swim times were significantly faster when compared to a placebo. Blood $\mathrm{pH}$ and bicarbonate concentrations were also significantly elevated post-bicarbonate ingestion. Siegler et al. (2007) found similar blood pH and bicarbonate increases while supplementing on sodium bicarbonate during cycling at supramaximal effort (53). Douroudos et al. (2006) also examined the effects of sodium bicarbonate ingestion on a maximal effort performance. The researchers found that subjects who ingested sodium bicarbonate significantly increased mean power output while a placebo showed no effect. The improvement in overall power output was attributed to sodium bicarbonate's ability to induce a more alkaline $\mathrm{pH}$ by increasing blood bicarbonate levels (16). This supports the idea that ingestion of sodium bicarbonate may enhance our acid-base balance system and therefore improve high-intensity performance.

More conclusive research on sodium bicarbonate's ergogenic potential can be observed during repeated intermittent sprints bouts (44). Early research by Costill et al. (1984) investigated the effects of sodium bicarbonate on multiple bouts of cycling performance. It was found that cycling performance times were significantly lower in subjects that supplemented on sodium bicarbonate during the last bout of exercise. The researchers also revealed that resting 
blood $\mathrm{pH}$ increased on average from 7.34 to 7.41 while bicarbonate levels rose from 27.5 to 31.0 $\mathrm{mm}$ in the sodium bicarbonate group. These levels were all significantly higher than the placebo group. This was one of the first studies that showed that sodium bicarbonate supplementation, by means of increasing $\mathrm{pH}$ and bicarbonate levels, can enhance performance.

Similarly, research conducted by Bishop et al. (2004-2005) found that an ingestion of sodium bicarbonate significantly improved power output and total work in repeated sprint settings. In the initial study (2004), the researchers used recreational sport participants to perform a series of five short (6-second) sprints with a recovery of 30 seconds. Similar to previous studies, lower blood concentrations of hydrogen ions $(\mathrm{H}+)$ and higher bicarbonate levels after sodium bicarbonate supplementing was noted (8). Although no difference was reported in total work or percent fatigue, improvements in sprints three through five occurred (8). This increase in performance in sprint 3-5 may be attributed to the higher levels of bicarbonate allowing for removal of hydrogen ions during the 30 -second rest period (8).

Lavender \& Bird (1989) also examined sodium bicarbonate supplementation on repeated sprints. Using a repeated and modified 10-second Wingate protocol, the authors found that the bicarbonate trials showed significantly greater mean power output compared to the placebo trials in during 8 out of 10 repeated ten-second cycle sprints.

Sodium bicarbonate ingestion has also been shown to significantly increase time to exhaustion (65). In a study comparing 4 buffering agents: bicarbonate, citrate, chloride, and lactate, Van Montfoort et al. (2004) found that sodium bicarbonate proved to be most effective in increasing time to exhaustion and reduced fatigue. This study, combined with previous research on benefits in power output, suggest that sodium bicarbonate may enhance sprints performance factors such as power output and fatigue. 
Although many studies have shown that sodium bicarbonate ingestion modifies the blood acid-base balance, its effects on performance are not always positive (20, 29, 30, 62). Previous studies failed to find improvements in 2-minute sprint bouts (29), peak power during a Wingate assessments (30), 600 meter run times (62), and repeated sprint bouts (20). In regards to repeated sprint bouts, there is a trend of research suggesting that the initial sprint of multiple sprint trials shows no significant improvement $(8,32)$. This lack of improvement in single shorts sprints may be attributed to a single sprint is too short to initiate extracellular buffering mechanisms. However, the repetitive nature of multiple sprints may facilitate greater buffering capacity over time (51). This suggests that sodium bicarbonate supplementation may benefit sprint performance measures such as peak power, fatigue, and feelings of fatigue during latter sprints.

Sodium bicarbonate has also shown to have no effect on endurance performance (22) as well as strength performance $(49,66)$. Stephens et al. (2002) found that even though subjects maintain small muscle and blood alkalosis during exercise, there were no benefits to the time taken to complete required work in endurance cycling. Portington et al. (1998) and Webster et al. (1993) found no effect of sodium bicarbonate supplementation on total work performed on 1 repetition maximum during leg press exercise. These researchers suggested that a slower rate of lactate and hydrogen ion $(\mathrm{H}+)$ production in resistance training, in contrast with other high intensity exercise bouts, minimizes the possible benefits of increased buffering capacity $(49,66)$.

Taken together, these studies suggest that:

- Sodium Bicarbonate supplementation increases high intensity performance, most notably, in intermittent or repeated sprints.

- Sodium Bicarbonate has no significant effect on aerobic exercise performance. 
- The primary determinants of increases in performance may be attributed to the increased buffering capacity of hydrogen ions through increased bicarbonate stores and lower $\mathrm{pH}$ levels resulting from creatine supplementation.

\section{Sodium Bicarbonate: Dosing regimens}

Current research suggests that sodium bicarbonate improves performance, however, there are various methods of supplementing with sodium bicarbonate. Two popular modes of administering sodium bicarbonate ingestion are acute and chronic supplementation. Most research has used the acute method which involves a one time loading dose of $0.3 \mathrm{~g} / \mathrm{kg}$ approximately $60-90$ minutes prior to exercise $(8,16,32,34,40,41,43,45,53,57,65)$. This dose equates to about 24 grams of sodium bicarbonate for an $80 \mathrm{~kg}$ male athlete (9). This has been considered the "traditional" method of sodium bicarbonate ingestion and is the minimum amount to see positive benefits on performance. This method, however, has shown to produce gastrointestinal side effects such as nausea, vomiting, and stomach cramping during exercise (40, $43,65)$.

The chronic method involves loading a larger dose of $0.5 \mathrm{~g} / \mathrm{kg}$ of bicarbonate over several days (9). This chronic loading method, for many athletic competitions, may provide more of a benefit than an acute, one time, loading dose. It is thought, but not fully understood, that due to changes in $\mathrm{pH}$ values and the bodies bicarbonate levels during the chronic loading period that the body manages to store the extra bicarbonate provided over a period of 5-6 days and can then use the extra buffer to improve performance (39). This method of ingestion has also shown to reduce gastrointestinal side effects almost completely. 
A study by McNaughton \& Thompson (2001) investigated the effects of acute versus chronic ingestion methods of sodium bicarbonate on anaerobic work and power output. In this study, subjects were randomized into an acute supplementation or chronic supplementation group. The acute supplementation group ingested $0.3 \mathrm{~g} / \mathrm{kg} 90$ minutes before exercise the exercise test, whereas the chronic group, ingested $0.5 \mathrm{~g} / \mathrm{kg}$ /day for 6 days and nothing on test day. A second performance test and blood analysis was also administered 2 days after the supplementation had ceased. Results from this study revealed that the acute and chronic group showed similar performance benefits. However, the chronic supplemented group significantly maintained improved performance two days after the supplementation ceased. Blood $\mathrm{pH}$ and bicarbonate levels were also not different from the first 24 hours of ingestion (43). Furthermore, the chronic supplementation group experienced no gastrointestinal side effects, whereas the acute supplemented group did. The similar performance and blood measure results suggest that chronic loading may be a more feasible and effective method of ingestion than acute supplementation. The authors concluded that since the positive benefits from chronic sodium bicarbonate supplementation lasted 2 days after supplementation ceased, a supplementation of less that 6 days may be just as effective and more feasible (43).

\section{Sodium Bicarbonate: Mechanism of action}

Sodium bicarbonate may aid in the acid-base balance system by buffering metabolites resulting from high intensity exercise. During high-intensity exercise, specifically after the utilization of PCr has ceased, energy needs are provided primarily by anaerobic glycolysis (51). In this energy system, the end product of glycolysis, pyruvic acid, is converted to lactic acid, 
which eventually recreates glucose for energy (36). In this process, lactic acid dissociates into a lactate molecule and a free hydrogen ion $\left(\mathrm{H}^{+}\right)$.

Glycolysis $\rightarrow$ pyruvic acid $\rightarrow$ lactic acid $+\left(\mathrm{H}^{+}\right)$

Increased $\mathrm{H}^{+}$concentrations result in decreased blood and muscle $\mathrm{pH}$ levels making the muscle and blood more acidic. Recent research suggests that the capability to sustain high-intensity exercise depends largely on the ability to minimize increases in these hydrogen ion concentrations $\left(\mathrm{H}^{+}\right)(52)$. Previous studies have concluded that the increase in hydrogen ions $(\mathrm{H}+)$ and a decrease in $\mathrm{pH}$ hinder aspects of performance such as power output and fatigue by a disruption of contractility within the muscle $(32,52)$. Specifically, studies have shown that increased hydrogen ions $(\mathrm{H}+)$ in blood and muscle may inhibit calcium release from the sarcoplasmic reticulum as well as inhibit the interaction between actin and myosin $(40,51)$.

This process, however, stimulates extracellular buffering mechanisms of which bicarbonate is one of the most effective agents (36). There are 4 basic mechanisms to alter blood and muscle $\mathrm{pH}$. First, bicarbonate can immediately buffer the hydrogen ions within seconds. Second, lactate produced from high intensity energy metabolism diffuses into the blood stream while shuttling a hydrogen ion $(\mathrm{H}+)$ out of the muscle cell. Third, bicarbonate then excretes hydrogen ions through ventilation. In response to this accumulation, bicarbonate binds with the free hydrogen ions $(\mathrm{H}+)$ forming carbonic acid $(\mathrm{H} 2 \mathrm{CO} 3)$ and ultimately transports the remaining $\mathrm{CO} 2$ to the lungs where it and water are then exhaled and removed (44). Finally, the kidneys excrete hydrogen ions $(\mathrm{H}+)$ as fixed acid and work on a long-term basis to maintain acid-base balance.

Ingesting alkalinizing agents such as sodium bicarbonate, may aid this buffering system by increasing bicarbonate ions (HCO3) and therefore raising $\mathrm{pH}$ in the blood at the beginning, 
during, and after exercise $(11,37,38,40,51)$. However, intramuscular $\mathrm{pH}$ and bicarbonate levels remain relatively the same. In this respect, the mechanism by which the intake of sodium bicarbonate may enhance performance seems to be due to the increased buffering capacity outside the cell (51). Previous studies have confirmed that increased extracellular $\mathrm{pH}$ and higher bicarbonate levels, after ingestion of sodium bicarbonate, increase the removal of hydrogen ions $(\mathrm{H}+)$ and lactate from the muscle (51). These studies suggest that this process is the primary determinant for the improving aspects of performance such as power output, fatigue, and perception of fatigue (51).

Combined Creatine and Sodium Bicarbonate Supplementation: Ergogenic Potential Although there has been much research on the effect creatine monohydrate and sodium bicarbonate alone on performance, only one study has examined the combined effect of these supplements. Mero et al. (2004) found that combining creatine and sodium bicarbonate improved 100-meter swim performance. This study, however, had several limitations. The study examined swimming performance in which the findings may not by applicable to weight bearing performance. The study also did not compare the benefits of their combined supplementation of creatine and sodium bicarbonate to creatine or sodium bicarbonate alone. Thus, there is no way to quantify how much, if at all, the added effect of the combined supplementation actually had on swimming performance. Lastly, the supplementation procedures did not technically "combine" the supplements in one drink. Subjects supplemented for 6 days of creatine and, on the $7^{\text {th }}$ day, ceased creatine supplementation and ingested the full dose of sodium bicarbonate. Because of this method, along with no comparison to either supplement alone, there is no way of determining if the pre-race bicarbonate supplementation overshadowed the effects of the weeklong dose of creatine. 


\section{Combined Creatine and Sodium Bicarbonate: Mechanism of Action}

Considering the well-documented ergogenic benefits of creatine and sodium bicarbonate supplementation, along with the fact that creatine and sodium bicarbonate aid in separate metabolic pathways, combining these two supplements may yield greater performance benefits when compared to either supplement alone. This hypothesis can be supported considering each supplement's mechanism of action (Figure 3 below). Creatine has the ability to rapidly regenerate ATP during high intensity exercise through increases in free creatine and PCr stores. Furthermore, sodium bicarbonate has the ability to enhance buffering capacity and removal of hydrogen ions during high intensity exercise. Considering these mechanisms, combining these supplements may demonstrate greater performance benefits in high intensity exercise than either supplement alone.

\section{Conclusions}

Creatine monohydrate and sodium bicarbonate are among the most popular and widely used supplements in the realm of sports and exercise. This literature review has revealed that research suggests that both creatine and sodium bicarbonate can be used independently to enhance high-intensity performance. Creatine has shown to act as an energy reserve to regenerate ATP during muscle contraction. Previous research suggests that appropriate oral supplementation of 15-25 grams per day for 2-5 days of creatine monohydrate can benefit high intensity performance by means of accelerating ATP production from increases in free creatine and PCr stores. Similar conclusions can be made for the use of sodium bicarbonate supplementation. Previous research suggests that supplementing on sodium bicarbonate, alone, can enhance high intensity performance by assisting our buffering system through increased bicarbonate stores and $\mathrm{pH}$ levels. Considering that only one known study has examined the 
combined effect of these two supplements and the experimental limitations of that study, more research is necessary to further understand their combined effect. In conclusion, since creatine and sodium bicarbonate function in different metabolic pathways and have no known interactive effects, a combined supplementation of creatine with sodium bicarbonate may further improve sprint performance when compared to either creatine or sodium bicarbonate alone. 


\section{Chapter 3}

\section{Methods}

\section{Overview}

The purpose of this study was to determine whether a combined supplementation of creatine monohydrate and sodium bicarbonate would further enhance the well-document research of creatine supplementation alone on repeated sprint performance. A double-blinded crossover study design was used in which each subject served as his own control. All subjects completed 3 conditions, followed by a 3-week washout period between each condition: 1) placebo, 2) creatine, and 3) creatine combined with sodium bicarbonate. In the morning after each condition, peak power, relative peak power, mean power, relative mean power, fatigue index, perceptions of fatigue and GI distress, and blood analysis were assessed during 610 second repeated Wingate test.

\section{Subjects}

Thirteen healthy males between the ages of 18 and 30 were recruited from California Polytechnic State University. Seven of the 13 subjects who participated in the study were recreational triathletes who participated on the Cal Poly triathlon team. Subjects were recruited on a voluntary basis through e-mail and flyers to participate in this double-blind research study. The health of the subjects was assessed by a health history questionnaire. Inclusion criteria for this study included the following: (1) VO2 $\max >55 \mathrm{ml} / \mathrm{kg} / \mathrm{min}$, (2) engage in at least 5 hours/week of aerobic exercise, 3) no recent history of creatine monohydrate, sodium bicarbonate supplementation, or any other supplementation in the prior six months), (4) familiar with using a cycle ergometer, and (5) have no metabolic or chronic disease that would place the subject at risk of injury or interfere with performance. Subjects were informed of the study 
requirements, benefits, and risks of the study. Each subject gave verbal and written consent to participate in the study. This study was approved by the Human Subjects Committee at California Polytechnic State University.

\begin{tabular}{|l|l|}
\hline Variables & (n) $=\mathbf{1 3}$ \\
\hline Age $(\mathrm{yr})$ & $21.1 \pm 0.6$ \\
\hline Height $(\mathrm{in})$ & $69.8 \pm 0.7$ \\
\hline Weight $(\mathrm{kg})$ & $74.1 \pm 2.1$ \\
\hline BMI $\left(\mathrm{kg} \cdot \mathrm{m}^{-2}\right)$ & $23.5 \pm 0.4$ \\
\hline VO2 Max $\left(\mathrm{ml} \cdot \mathrm{kg}^{-1} \cdot \mathrm{min}^{-1}\right)$ & $66.7 \pm 5.7$ \\
\hline
\end{tabular}

Table 1. Subject Characteristics. Values are mean $( \pm \mathrm{SD})$.

Preliminary Tests

Subjects completed a Physical Activity Readiness Questionnaire (PAR-Q), a record of physical activity, and a health history questionnaire prior to participating in this study. Height, weight, body mass index (BMI), and age was assessed prior to the start of testing. To test for physical fitness, a graded exercise test (GXT) was administered to measure maximal oxygen consumption. The GXT was performed on a Monarch cycle ergometer and followed the Astrand Bicycle Ergometer Maximal Protocol. This protocol consisted of 3-minute stages while increasing the resistance on the cycle ergometer. The initial power output was $600 \mathrm{kgm} / \mathrm{min}(100$ W). After this initial stage, $50 \mathrm{~W}$ was added every 3 minutes. The test continued until 3 of the following 4 conditions were met: 1) $\mathrm{VO} 2>55 \mathrm{ml} \cdot \mathrm{kg}^{-1} \cdot \mathrm{min}^{-1}$; 2) respiratory exchange ratio > $1.15 ; 3)$ the subject's heart rate was within 5 beats of their age predicted max; and 4) the subject voluntarily stopped the test. During the graded exercise test, expired air was analyzed using an 
online system (Parvo Medics 2005, Salt Lake City, UT). VO2 Max was determined by the highest 30-second value obtained.

\section{Supplementation Procedure}

Using a double-blinded, crossover study design, subjects underwent each of the following conditions followed by a 3-week washout period: (1) placebo, 2) creatine alone, and 3) creatine + sodium bicarbonate (Table 2). Each of these conditions had a combination of two supplements to mask for the combined creatine and sodium bicarbonate supplementation. The placebo supplementation $(\mathrm{Pl})$ consisted of $20 \mathrm{~g}$ of maltodextrin combined with an additional $0.5 \mathrm{~g} / \mathrm{kg}$ of maltodextrin. The creatine alone supplementation (Cr) consisted of $20 \mathrm{~g}$ of creatine monohydrate combined with and additional $0.5 \mathrm{~g} / \mathrm{kg}$ of maltodextrin. The creatine + sodium bicarbonate supplementation $(\mathrm{CrSb})$ consisted of $20 \mathrm{~g}$ of creatine monohydrate combined with an additional $0.5 \mathrm{~g} / \mathrm{kg}$ of sodium bicarbonate. The addition of $0.5 \mathrm{~g} / \mathrm{kg}$ of maltodextrin to the placebo and creatine conditions was used to keep the volume consistent in each condition while also masking for the combined $\mathrm{Cr}+\mathrm{Sb}$ supplementation. This total daily dose was divided equally into 4 smaller doses each day to minimize gastrointestinal side effects. Each supplementation was ingested for 2 consecutive days. The supplements were unidentifiable looking and tasting which was determined after a pilot study taste test. On the first morning of each condition, subjects were given the supplements along with information on how and when to take the supplements (Appendix D). Subjects were instructed to ingest each supplement at the following times: 9:00 a.m., 12:00 p.m., 6:00 p.m., and 10:00 p.m. Subjects were instructed to drink at least 16 ounces of water during each ingestion and at least 2 liters of water throughout each supplementation day. Subjects completed a 24-hour dietary recall and were instructed to keep caloric intake similar in each condition. Subjects were also instructed to refrain from caffeine, alcohol, and exercise 
during the supplementation period. Caffeine and alcohol were excluded to because they are drugs that effect performance. Finally, subjects were instructed to fast during the morning of the repeated Wingate test. Subjects were asked to sign a confirming that they adhered to the supplementation and dietary recall instructions.

\begin{tabular}{|c|c|c|c|c|}
\hline & Day 1 & Day 2 & $\begin{array}{c}\text { Day 3 } \\
\text { Repeated Wingate Test }\end{array}$ & $\begin{array}{c}\text { Washout } \\
\text { Period }\end{array}$ \\
\hline Treatment \#1 & Placebo & Placebo & None & 3 weeks \\
\hline Treatment \#2 & $\mathrm{Cr}$ & $\mathrm{Cr}$ & None & 3 weeks \\
\hline Treatment \#3 & $\mathrm{Cr}+\mathrm{Sb}$ & $\mathrm{Cr}+\mathrm{Sb}$ & None & 3 weeks \\
\hline
\end{tabular}

Table 2. Supplementation Model. All subjects received each of the following treatments using a double-blind, counter-balanced, crossover design.

\section{Repeated Sprint Test}

On the third morning of the supplementation period, subjects performed a modified repeated Wingate test. The modified protocol was similar to the one conducted by Ziegenfuss et al. (2002). This consisted of six 10 -second Wingate sprints each followed by a 60 -second active rest. The sprint test procedures started with a 5-minute warm-up at 50-150 $\mathrm{W}$ with three 5second practice sprints. At the end of this warm-up, the test administrator gave a 10-second countdown for the subject to build up to their maximal effort. At the end of this countdown, the cycle ergometer magnetically added $0.075 \mathrm{~kg}$ per kilogram of body weight for resistance immediately at the start of the sprint. Subjects were instructed to perform maximally throughout each 10 -second sprint while verbal encouragement was administered. Immediately following each 10 -second sprint, a 60 -second active rest at $50 \mathrm{~W}$ was performed. This procedure was repeated 6 times. All Wingate assessments were performed on the Velotron Dynafit Pro cycle 
ergometer (RaceMate Inc, Seattle, WA). Test administrators recorded optimal seat-heights for each subject (the seat height was kept the same for each subject on each test).

The performance measures that were assessed during the Wingate test were: (1) peak power (W); (2) relative peak power (W/kg); (3) mean power (W); (4) relative mean power (W/kg); and (5) Fatigue Index (W/S). All dependant measures were recorded on the corresponding Racermate Velotron Wingate Software.

Subjective measures included: perceived feelings of fatigue, gastrointestinal distress and systemic discomfort. Perceived feelings of fatigue were assessed using a 0-10 ratings scale provided in the Multidimensional Fatigue Questionnaire. Gastrointestinal distress was assessed using the same questions used in a study by Pfeiffer et al. (2009). The questionnaire addressed upper abdominal problems (e.g. bloating, cramps, vomiting, nausea) and lower abdominal problems (intestinal cramps, flatulence, and diarrhea). Systemic discomfort was assessed by rating any systemic problems (dizziness, headache, muscle cramp, or urge to urinate). Each question was rated on a 0 - 10 point scale ranging from $0=$ no problem at all to $10=$ the worst it has ever been.

\section{Blood Collection and Analysis}

Blood capillary samples were administered via finger prick. Capillarized blood samples were taken from the finger by using a sterile lancet. All blood samples will be directly recorded on an Abbott iSTAT Blood Gas Analyzer (Version 1 2006, Abbot Park, IL). The blood analyzer was calibrated prior to and at the end of the test session. Blood samples were recorded 5 minutes before the first Wingate sprint and 5 minutes after the sixth Wingate sprint. The dependant measures recorded from the blood sample were: blood $\mathrm{pH}$, bicarbonate $(\mathrm{mmol} / \mathrm{L})$, and lactate $(\mathrm{mmol} / \mathrm{L})$. 


\section{Statistical Analysis}

Statisitcal Analysis Software Inc. (SAS Institute, Cary, North Carolina) was used for statistical analysis of the data in this study. A repeated measures ANOVA was used to determine condition, sprint, and condition by sprint interactions for peak power, relative peak power (RPP), mean power, relative mean power (RMP), Fatigue Index (FI), perceptions of fatigue and GI distress, and the blood analysis. Due to the crossover nature of the design, each subject underwent each treatment allowing comparisons of treatment means to be done 'within subject'. Because each subject will underwent six sprints (repeated measurements) after application of each condition, the effect of condition and the condition by sprint interaction was evaluated 'within subject' and a Tukey's post hoc analysis was administered.

\section{Sample Size Calculation}

Relative peak power output (RPP) data from Ziegenfuss et al. (2002) was used to calculate the sample size for this study. This study replicated the testing protocol used by Ziegenfuss et al. (2002). Power computations were carried out using a standard deviation of 0.75 $\mathrm{W} / \mathrm{kg}$ to detect a difference of $1 \mathrm{~W} / \mathrm{kg}$ with $\alpha=0.05$ with power of 0.8 , which required a sample size of at least 12 subjects. 


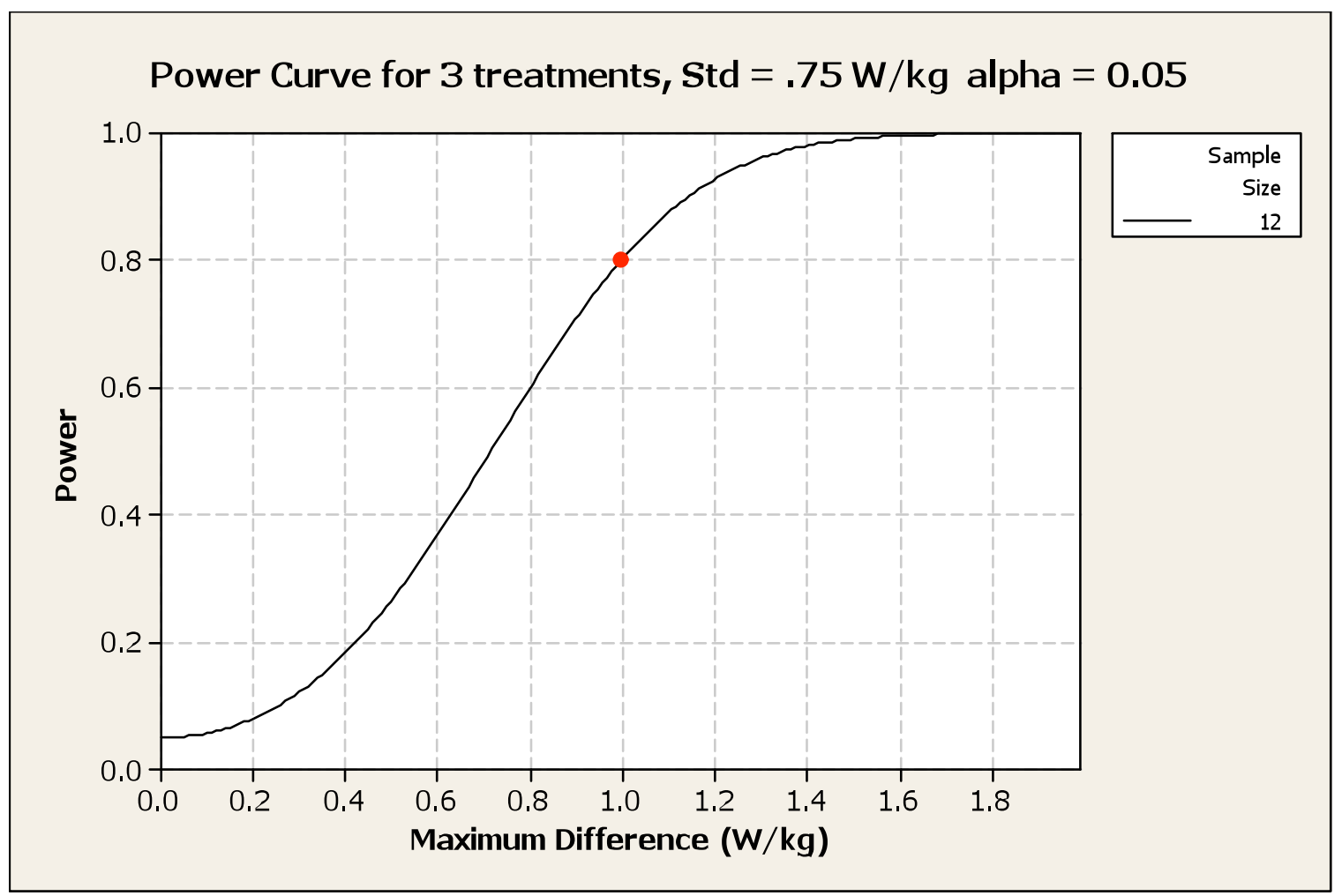

Figure 1. Power Curve for 3 treatments. $\mathrm{Std}=$ Standard Deviation, $\mathrm{W} / \mathrm{kg}=$ Watts per kilogram, Alpha $=$ Significance level.

\section{Pilot Study Data}

A pilot study was conducted prior to the start of data collection. Two subjects underwent 2 conditions followed by a one-week washout period. The two conditions were: 1$) \mathrm{Cr}+\mathrm{Sb}(20 \mathrm{~g}$ of creatine combined with $0.5 \mathrm{~g} / \mathrm{kg}$ of sodium bicarbonate) and 2) Cr only ( $20 \mathrm{~g}$ of creatine and $0.5 \mathrm{~g} / \mathrm{kg}$ of maltodextrin). These subjects supplemented on this dose in 4 smaller amounts throughout the day for 2 consecutive days. Pilot test subjects were instructed to ingest each supplement at the following times: 9:00 a.m., 12:00 p.m., 6:00 p.m., and 10:00 p.m. On the third day of each treatment, the subjects performed six 10-second repeated Wingate sprints on the cycle ergometer with a 60 -second rest in between each sprint. Both subjects were blinded to the treatment and had no prior history of supplementing on either creatine or sodium bicarbonate. 
Figures 2 and 3 displays the data for relative peak power over each sprint for each subject. Visually, it is apparent that both subjects were better able to attenuate the decline in power output over each sprint when supplementing on $\mathrm{Cr}+\mathrm{Sb}$. The greatest differences between treatments were observed during sprint 4,5 and 6.

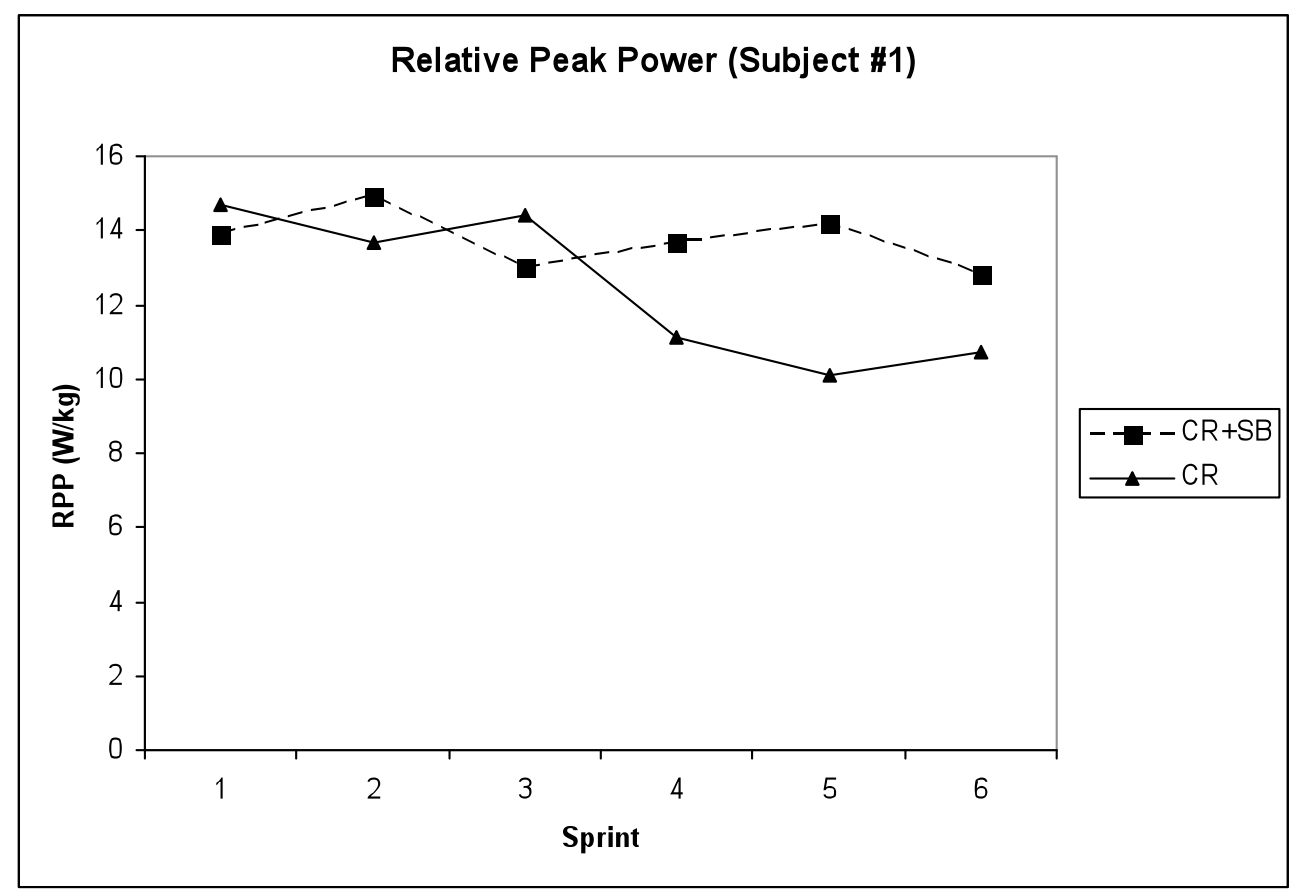

Figure 2. Pilot study data. Relative Peak Power values during the 6 repeated sprints in sample subject $\# 1$. The two conditions were $\mathrm{Cr}+\mathrm{Sb}=$ Creatine combined with sodium bicarbonate and $\mathrm{Cr}=$ Creatine alone. Values are in means. $\mathrm{W} / \mathrm{kg}=$ Watts per kilogram. 


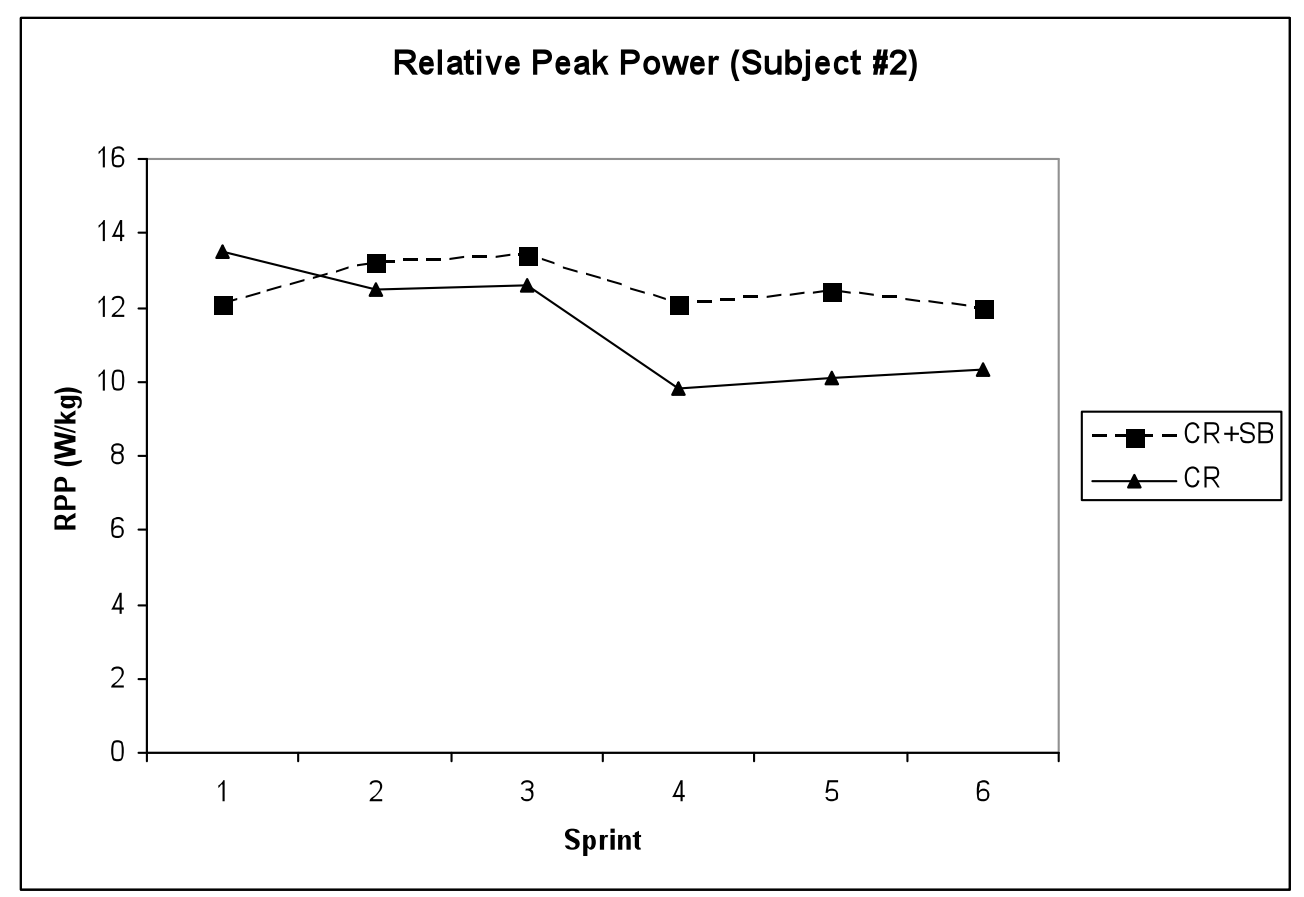

Figure 3. Pilot study data. Relative Peak Power values during the 6 repeated sprints in sample subject \#2. The two conditions were $\mathrm{Cr}+\mathrm{Sb}=\mathrm{Creatine}$ combined with Sodium Bicarbonate and $\mathrm{Cr}=$ Creatine alone. Values are in means. $\mathrm{W} / \mathrm{kg}=$ Watts per kilogram.

\section{Expected Results (Relative to placebo)}

It was expected that both creatine supplementation $(\mathrm{Cr})$ and combined creatine with sodium bicarbonate supplementation $(\mathrm{Cr}+\mathrm{Sb})$ would produce significantly higher peak and mean power values compared to the placebo $(\mathrm{P})$. However, it was expected that the $\mathrm{Cr}+\mathrm{Sb}$ supplementation would produce the greatest increase. It was expected that both $\mathrm{Cr}$ supplementation and $\mathrm{Cr}+\mathrm{Sb}$ supplementation will produce significantly lower fatigue index values (FI) and perceptions of fatigue ratings when compared to the placebo, with the $\mathrm{Cr}+\mathrm{Sb}$ supplementation showing the greatest decrease. Finally, $\mathrm{Cr}$ supplementation and $\mathrm{Cr}+\mathrm{Sb}$ supplementation will show significantly higher blood bicarbonate, $\mathrm{pH}$, and lactate levels throughout the exercise test, with the $\mathrm{Cr}+\mathrm{Sb}$ supplementation yielding the highest values. 


\begin{tabular}{|c|c|c|c|c|c|}
\hline & $\begin{array}{c}\text { Relative } \\
\text { Peak Power } \\
\text { (RPP) }\end{array}$ & $\begin{array}{c}\text { Relative } \\
\text { Mean Power } \\
\text { (RMP) }\end{array}$ & $\begin{array}{c}\text { Fatigue } \\
\text { Index } \\
\text { (FI) }\end{array}$ & $\begin{array}{l}\text { Perceptions } \\
\text { of Fatigue }\end{array}$ & $\begin{array}{c}\text { Blood } \\
\text { bicarbonate, } \\
\text { pH, and } \\
\text { lactate }\end{array}$ \\
\hline $\begin{array}{c}\text { CR } \\
\text { Supplementation }\end{array}$ & $\begin{array}{l}\Uparrow \text { In all } 6 \\
\text { sprints }\end{array}$ & $\begin{array}{l}\Uparrow \text { In all } 6 \\
\text { sprints }\end{array}$ & $\begin{array}{c}\Downarrow \text { In all } 6 \\
\text { sprints }\end{array}$ & $\begin{array}{c}\Downarrow \text { In all } 6 \\
\text { sprints }\end{array}$ & $\begin{array}{c}\Uparrow \text { In all } 6 \\
\text { sprints }\end{array}$ \\
\hline $\begin{array}{c}\text { CR + SB } \\
\text { Supplementation }\end{array}$ & $\begin{array}{c}\Uparrow \Uparrow \text { In all } 6 \\
\text { sprints }\end{array}$ & $\begin{array}{c}\Uparrow \Uparrow \text { In all } 6 \\
\text { sprints }\end{array}$ & $\begin{array}{c}\Downarrow \Downarrow \text { In all } 6 \\
\text { sprints }\end{array}$ & $\begin{array}{c}\Downarrow \Downarrow \text { In all } 6 \\
\text { sprints }\end{array}$ & $\begin{array}{c}\Uparrow \Uparrow \text { In all } 6 \\
\text { sprints }\end{array}$ \\
\hline
\end{tabular}

Table 3. All are in relation to placebo. $\Uparrow=$ Increase, $\Uparrow \Uparrow=$ Greater increase $\Downarrow$ Decrease $\Downarrow \Downarrow$ Greater Decrease

\section{Limitations}

- Measuring Sprint Performance: The Wingate Anaerobic Power test was used for measuring relative peak power (RPP), relative mean power (RMP), and fatigue index (FI). In this test, maximal effort may vary from each subject, which may underestimate peak power values and may cause confounding interpretation of results. All subjects, however, will be instructed to give maximal effort throughout the entire sprint trial and will be given verbal encouragement.

- Blood Analysis: Due to lack of funds, every subject did not take the blood analysis.

However, 10 randomly selected subjects underwent this assessment for each condition. Blood samples were only measured at 5 minutes before the first Wingate and 5 minutes after the last Wingate test. 
- Adherence: Participants were expected to adhere to the dosing protocol through the consumption of each supplement or placebo. Subjects were required to return the supplementation containers to the laboratory for refilling. Subjects signed a contract each day confirming that they adhered to this protocol.

- Gastrointestinal side effects: Sodium bicarbonate ingestion has been shown to produce gastrointestinal side effects during exercise. Feelings of nausea, vomiting, or stomach cramping can potentially hinder a subject's maximum effort and/or performance.

However, to reduce these symptoms, subjects ingested each supplementation in 4 smaller doses at 9:00 a.m., 12:00 p.m., 6:00 p.m., and 10:00 p.m. throughout the ingestion period and fasted on test day. 


\section{Chapter 4}

\section{$\underline{\text { Results }}$}

\section{Performance Measures}

In this study, no significant sequence or period effect was observed. In addition, no carryover effect was observed demonstrating that the 3-week washout period was sufficient.

\section{Peak Power Output}

Performance data from all Wingate results is presented in Table 4. A main effect for condition was observed for peak power output $(\mathrm{p}=0.0284)$. Peak power values for the combined creatine and sodium bicarbonate condition were significantly higher than the placebo condition (Figure 4.1). There was no significant difference in peak power values for the creatine alone condition and the combined creatine and sodium bicarbonate condition. There was no significant difference between the creatine alone condition and the placebo condition.

A main effect for time was observed for peak power $(\mathrm{p}<0.001)$ Time was represented by each of the repeated sprint trials. Each condition showed significantly lower power output values in sprint 4, 5, and 6 when compared to sprint 1 (Table 4). No significant interaction of condition $\mathrm{x}$ time was observed $(\mathrm{p}=0.3158)$. 


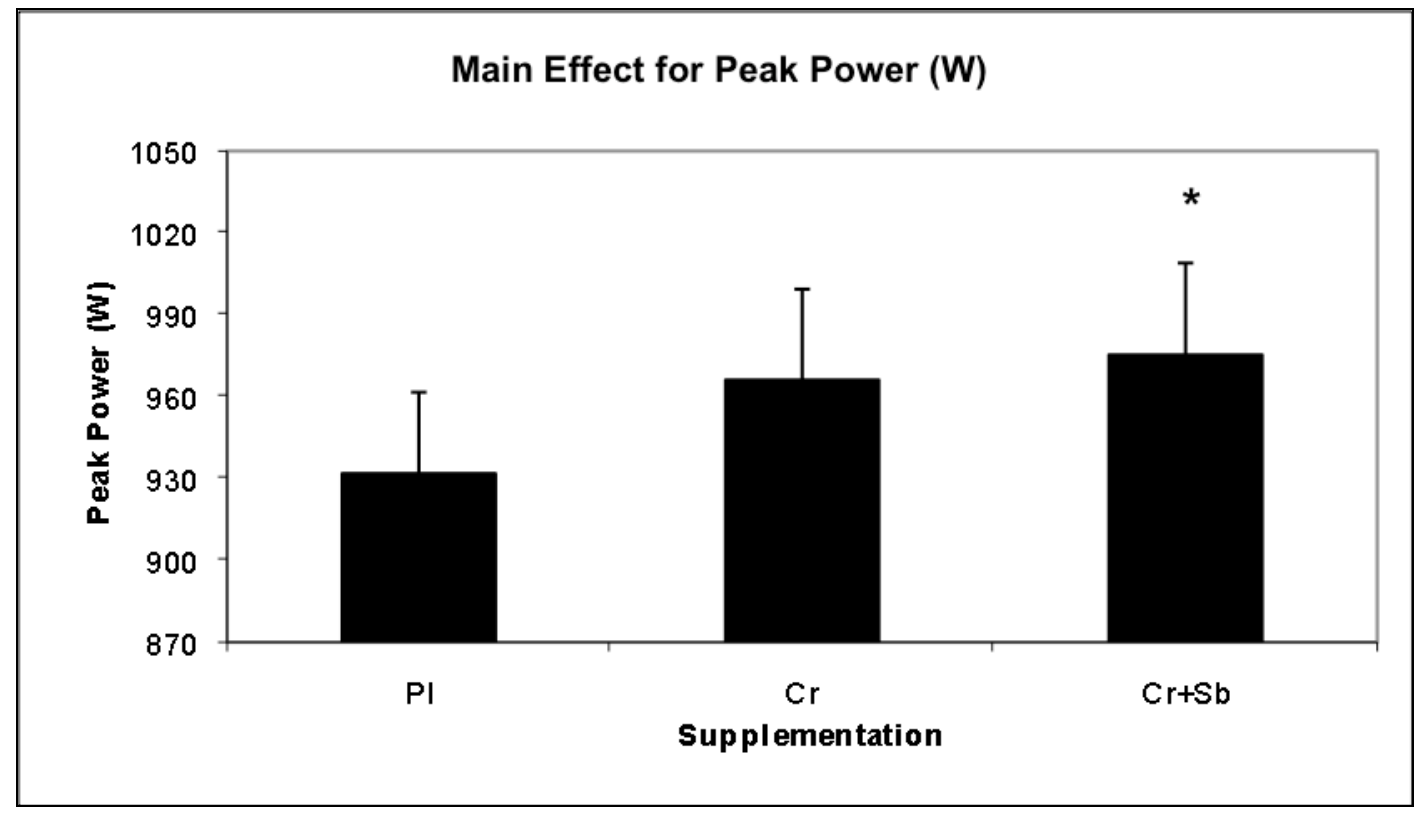

Figure 4.1. Main effect chart for peak power during the six repeated Wingate sprints in response to each condition. Values are in means $\pm \mathrm{SEM} ; \mathrm{W}=$ Watts, $\mathrm{Pl}=$ placebo, $\mathrm{Cr}=$ creatine, $\mathrm{Cr}+\mathrm{Sb}=$ creatine and sodium bicarbonate. ${ }^{*} \mathrm{Cr}+\mathrm{Sb}$ significantly higher than placebo $(\mathrm{P}=0.0302)$

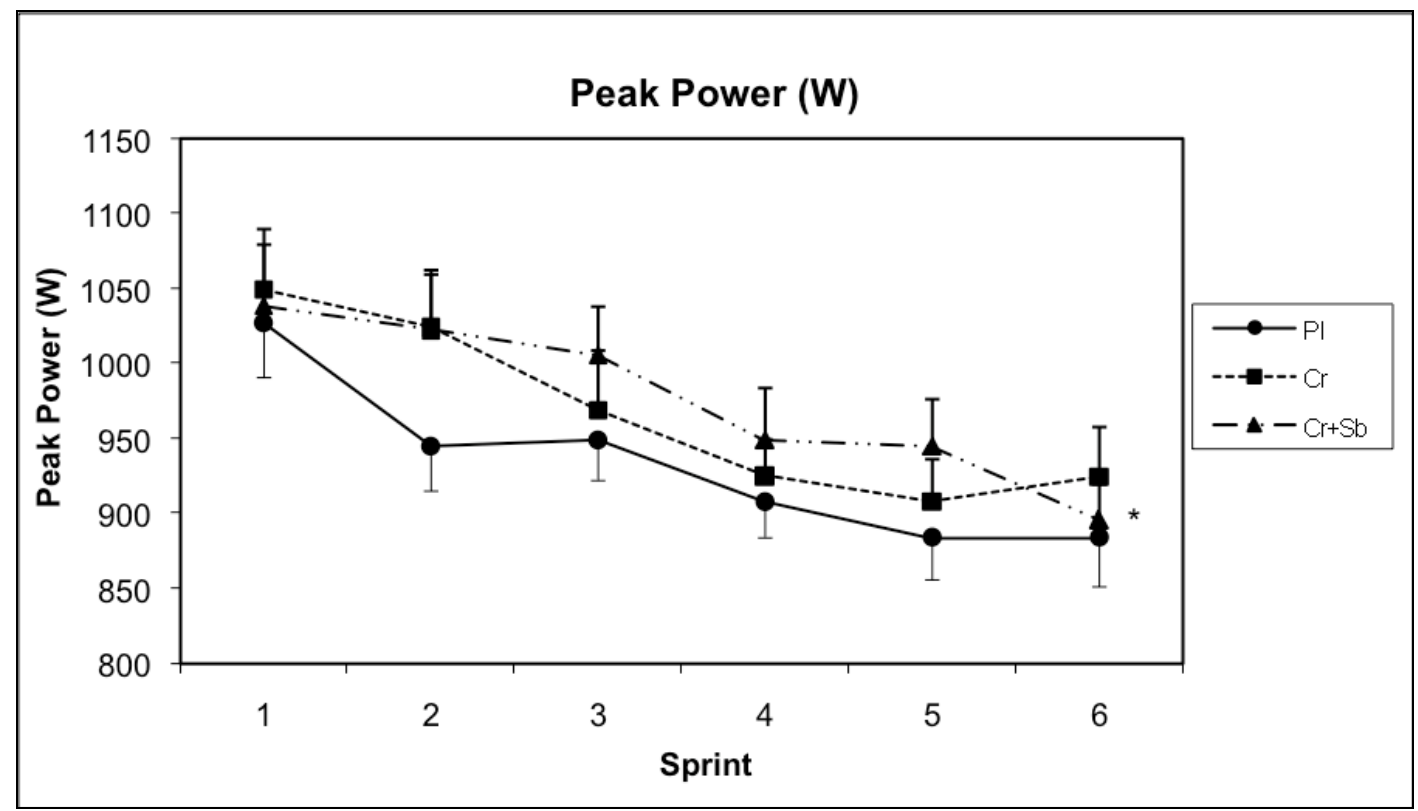

Figure 4.2. Peak power values during the 6 repeated sprints in response to each condition. Values are in means $\pm \mathrm{SEM} ; \mathrm{W}=$ Watts. $\mathrm{Pl}=$ placebo, $\mathrm{Cr}=$ Creatine, $\mathrm{CrSb}=$ creatine and sodium bicarbonate. ${ }^{*} \mathrm{Cr}+\mathrm{Sb}$ significantly higher than placebo $(\mathrm{P}=0.03)$ 


\section{Relative Peak Power}

For relative peak power $(\mathrm{W} / \mathrm{kg})$, a main effect for condition was observed $(\mathrm{p}=0.0023)$. Relative peak power values for the creatine alone condition and the combined creatine and sodium bicarbonate condition were significantly higher than the placebo condition (Figure 5.1). The creatine alone condition was not significantly different from the combined creatine and sodium bicarbonate condition $(\mathrm{p}=0.5279)$.

A main effect for time was observed for relative peak power $(p<0.0001)$. In the placebo condition, relative peak power values in sprints 4,5 and 6 showed to be significantly different from sprint 1 (Table 4, page 42). In the creatine alone condition, relative peak power values in sprints 4,5 , and 6 showed to be significantly different from sprint $1(\mathrm{p}<0.0001)$. However, in the combined supplementation condition, the only relative peak power value different from sprint 1 was observed in sprint 5 (Table 4). There was no significant interaction found for condition $\mathrm{x}$ time $(\mathrm{p}=0.4730)$. 


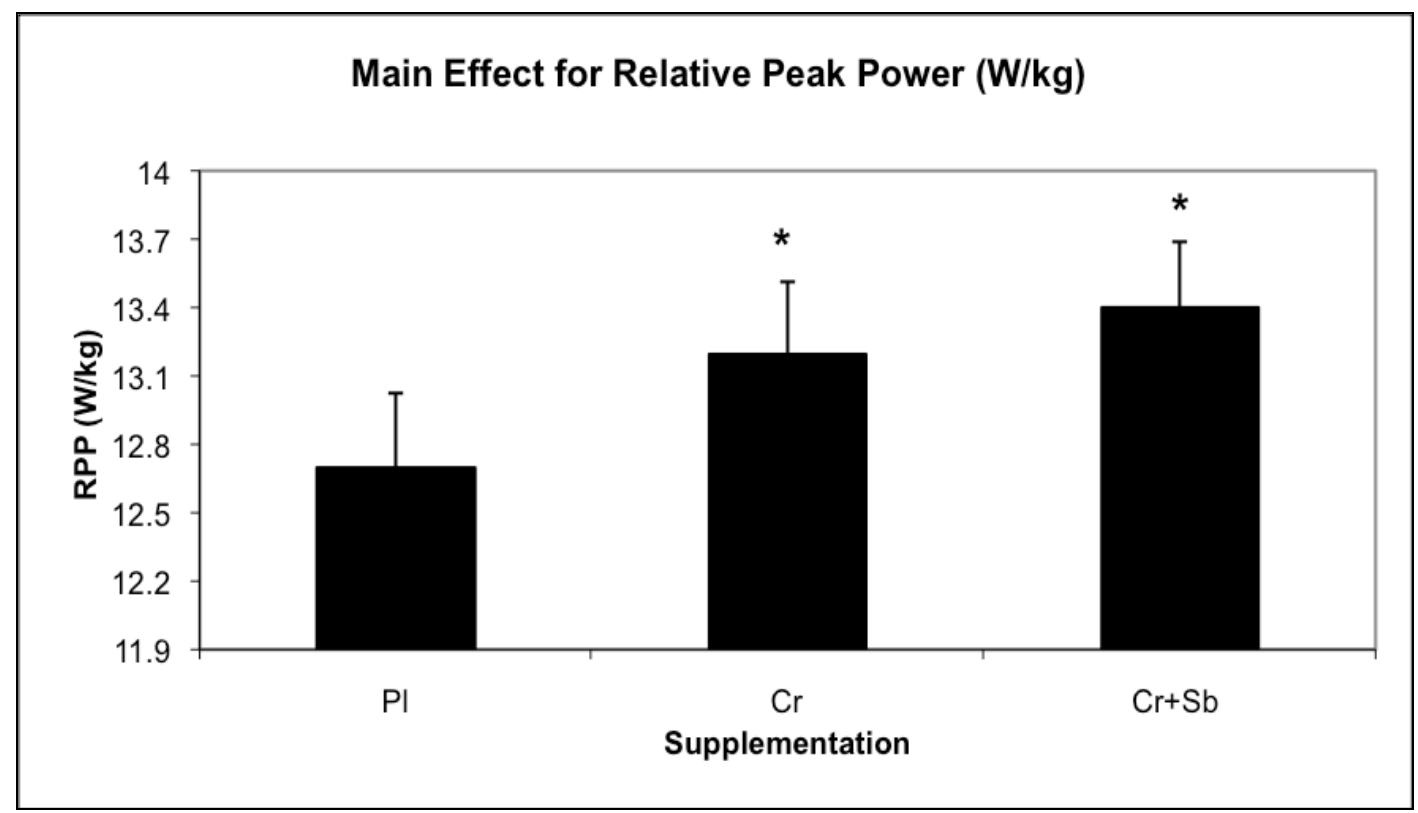

Figure 5.1 Main effect chart for relative peak power during the six repeated sprints in response to each condition. Values are in means $\pm \mathrm{SEM} ; \mathrm{W} / \mathrm{kg}=$ Watts per kilogram. $\mathrm{Pl}=$ placebo, $\mathrm{Cr}=$ Creatine, $\mathrm{CrSb}=$ creatine and sodium bicarbonate. ${ }^{*} \mathrm{Cr}+\mathrm{Sb}$ significantly higher than placebo $(\mathrm{p}$ $=0.0021)$ and $\mathrm{Cr}$ significantly higher than $\mathrm{Pl}(\mathrm{p}=0.0261)$.

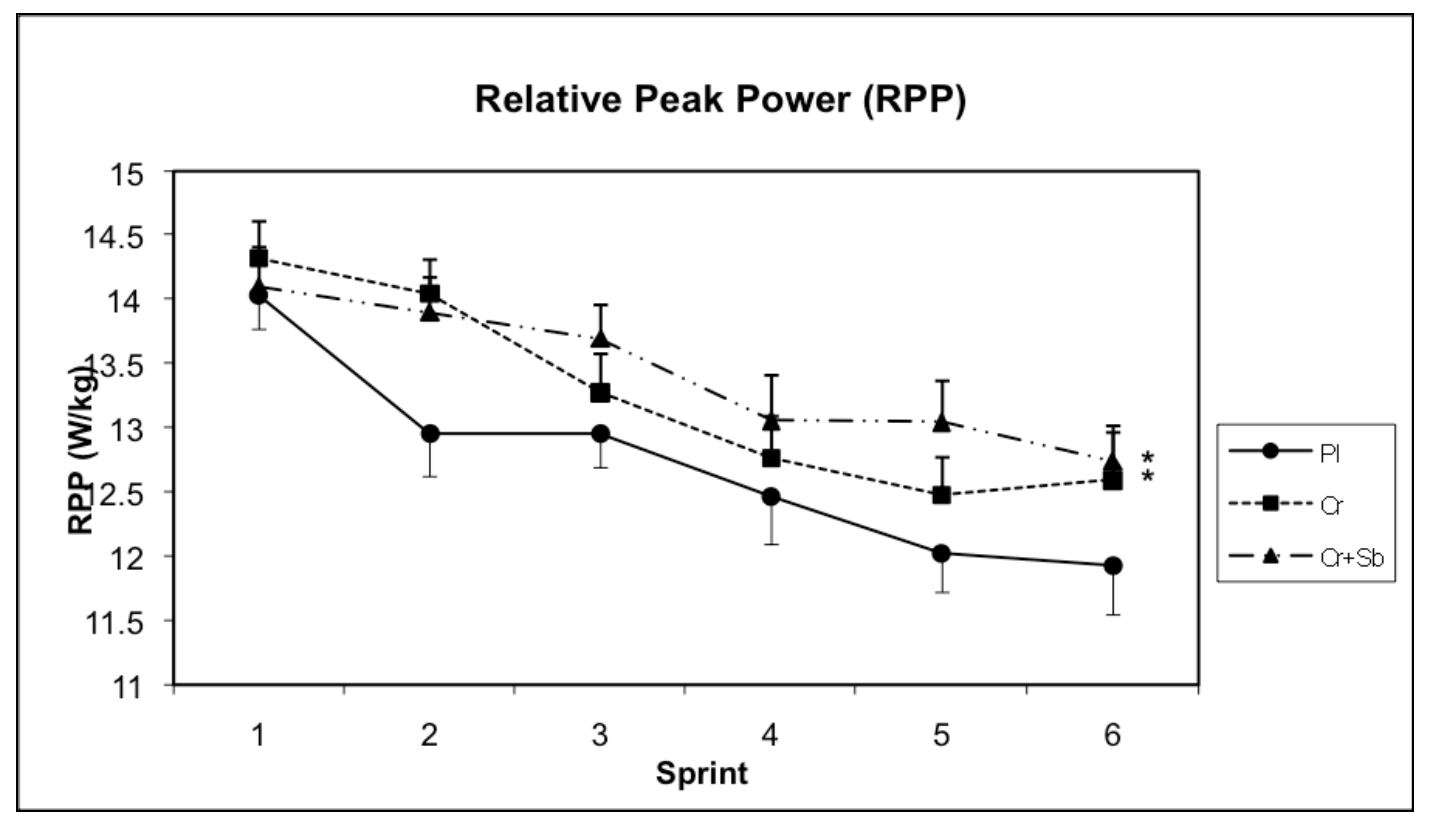

Figure 5.2 Relative peak power values during the 6 repeated sprints in response to each condition. Values are in means $\pm \mathrm{SEM} ; \mathrm{W} / \mathrm{kg}=$ Watts per kilogram. $\mathrm{Pl}=$ placebo, $\mathrm{Cr}=\mathrm{Creatine}$, $\mathrm{CrSb}=$ creatine and sodium bicarbonate. ${ }^{*} \mathrm{Cr}+\mathrm{Sb}$ significantly higher than placebo $(\mathrm{p}=0.0021)$ and $\mathrm{Cr}$ significantly higher than $\mathrm{Pl}(\mathrm{p}=0.0261)$. 


\section{Mean Power}

For mean power $(\mathrm{W})$, a main effect was observed $(\mathrm{p}=0.0267)$. Mean power values for the combined creatine and sodium bicarbonate condition were significantly higher than the placebo condition, however, no difference was found between the combined creatine and sodium bicarbonate condition and the creatine alone condition (Figure 6.1). The mean power values in the creatine alone condition were not significantly different from the placebo condition (Figure 6.1). There was no significant interaction found for condition $x$ time $(p=0.9933)$.

A time effect was observed for mean power $(\mathrm{p}<0.0001)$. Mean power values in sprints $3,4,5$, and 6 were significantly lower than in sprints 1 and 2 in all three conditions.

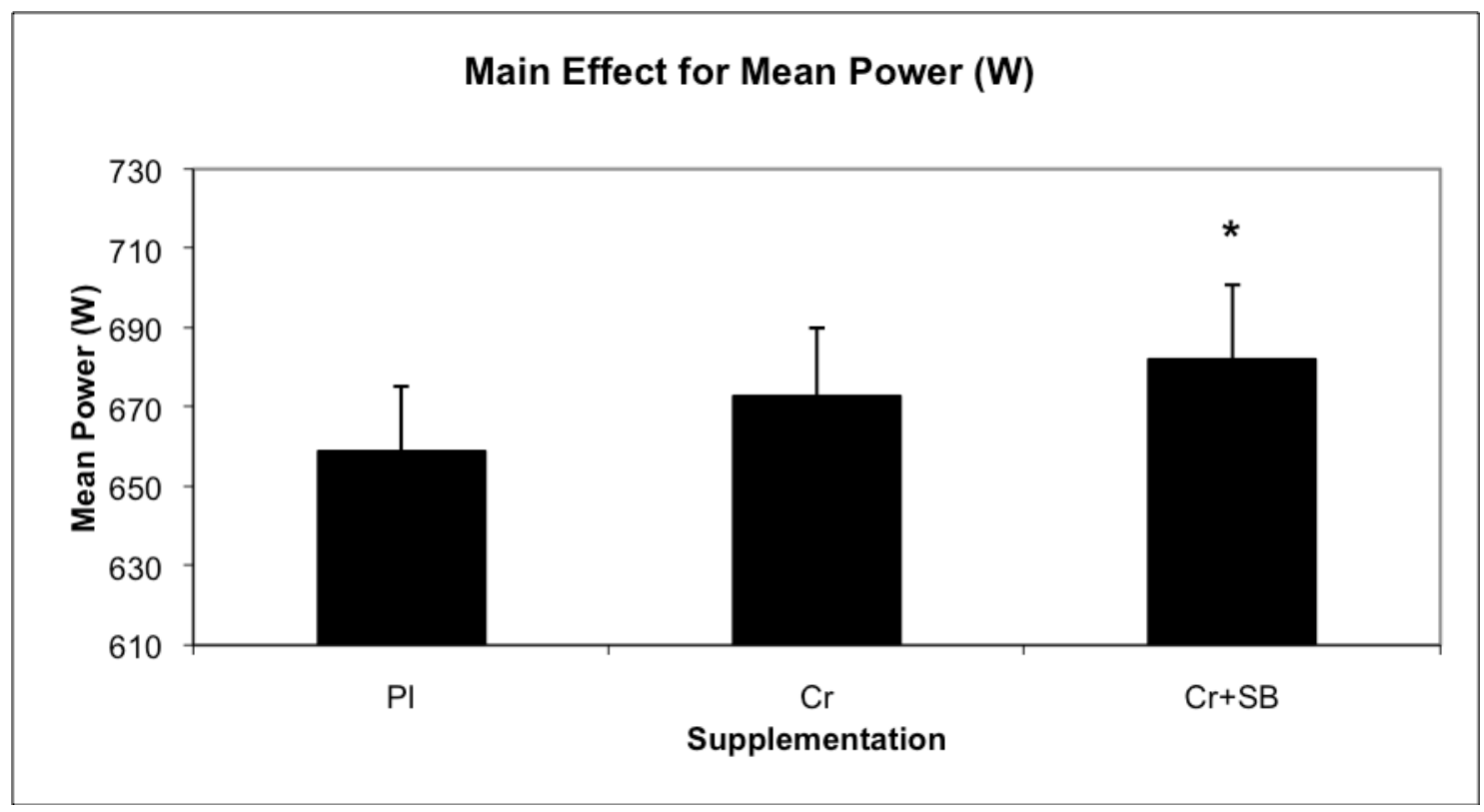

Figure 6.1 Main effect chart for mean power during the six repeated sprints in response to each condition. Values are in means $\pm \mathrm{SEM} ; \mathrm{W}=$ Watts per kilogram. $\mathrm{Pl}=$ placebo, $\mathrm{Cr}=$ Creatine, $\mathrm{CrSb}=$ creatine and sodium bicarbonate. ${ }^{*} \mathrm{Cr}+\mathrm{Sb}$ significantly higher than placebo $(\mathrm{p}=0.0208)$. 


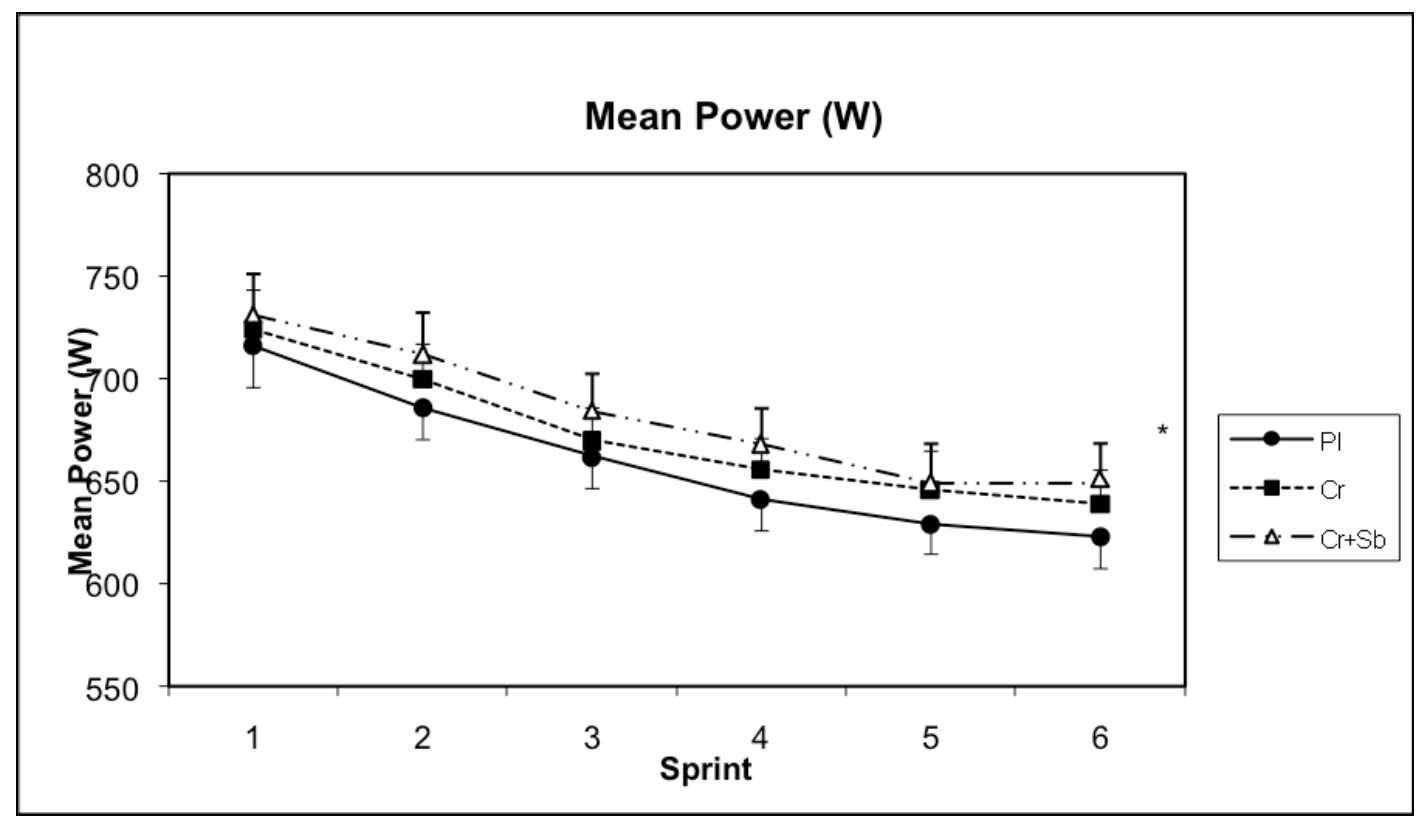

Figure 6.2 Mean power values during the 6 repeated sprints in response to each condition. Values are in means $\pm \mathrm{SEM}$; $\mathrm{W}=$ Watts. $\mathrm{Pl}=$ placebo, $\mathrm{Cr}=$ Creatine, $\mathrm{CrSb}=$ creatine and sodium bicarbonate. ${ }^{*} \mathrm{Cr}+\mathrm{Sb}$ significantly higher than placebo.

\section{Relative Mean Power}

For relative mean power $(\mathrm{RMP})$, no main effect was observed $(\mathrm{p}=0.1441)$. A time effect was observed $(\mathrm{p}<0.0001)$. There was no significant interaction found for condition $\mathrm{x}$ time $(p=0.9881)$ 


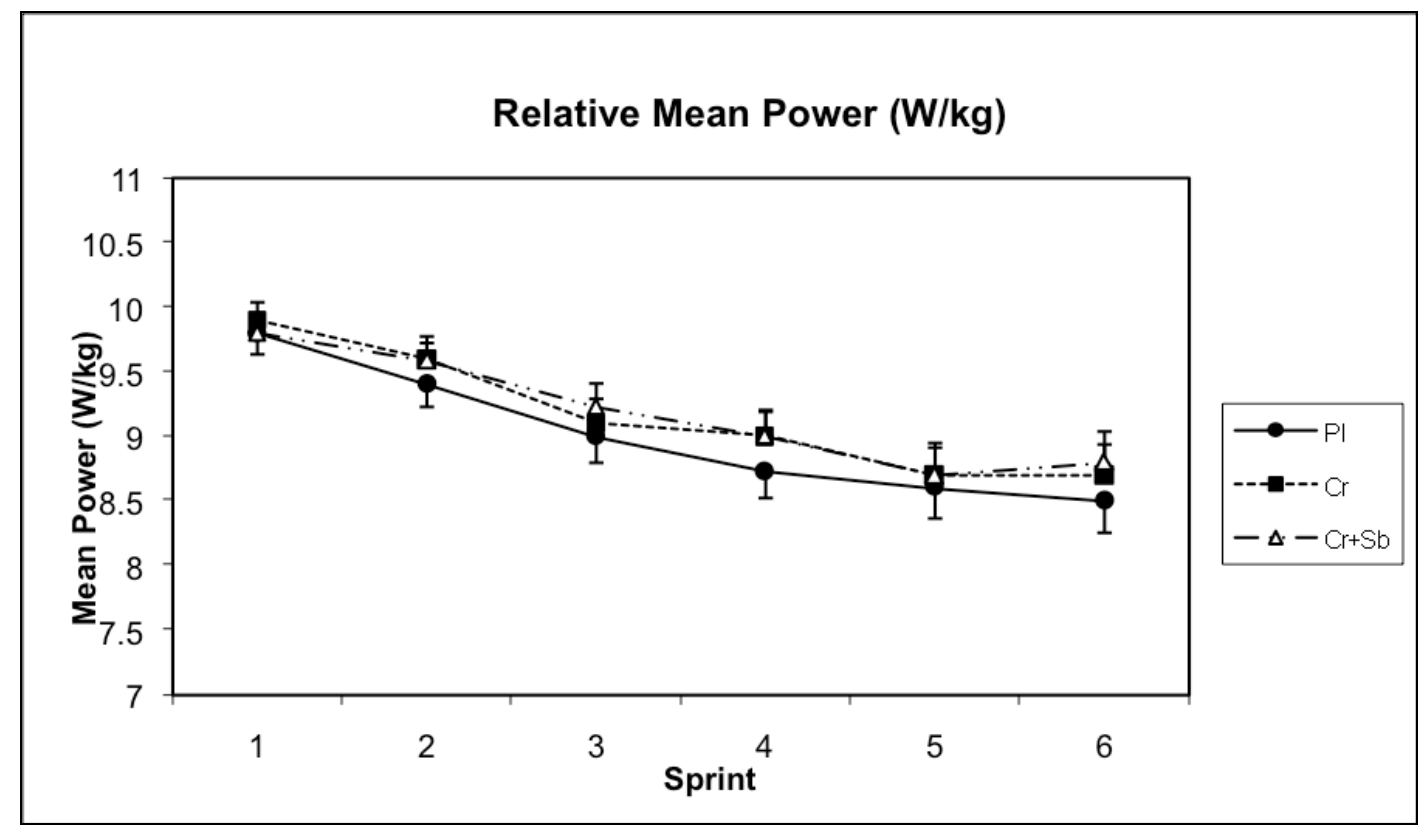

Figure 7. Relative mean power values during the 6 repeated sprints in response to each condition. Values are in means $\pm \mathrm{SEM} ; \mathrm{W} / \mathrm{kg}=$ Watts per kilogram. $\mathrm{Pl}=$ placebo, $\mathrm{Cr}=$ Creatine, $\mathrm{CrSb}=$ creatine and sodium bicarbonate.

\section{Fatigue Index}

For fatigue index $(\mathrm{FI})$, no main effect was observed $(\mathrm{p}=0.4240)$. A time effect was observed $(\mathrm{p}<0.0001)$. There was no significant interaction found for condition $\mathrm{x}$ time $(\mathrm{p}=$ $0.2938)$ 


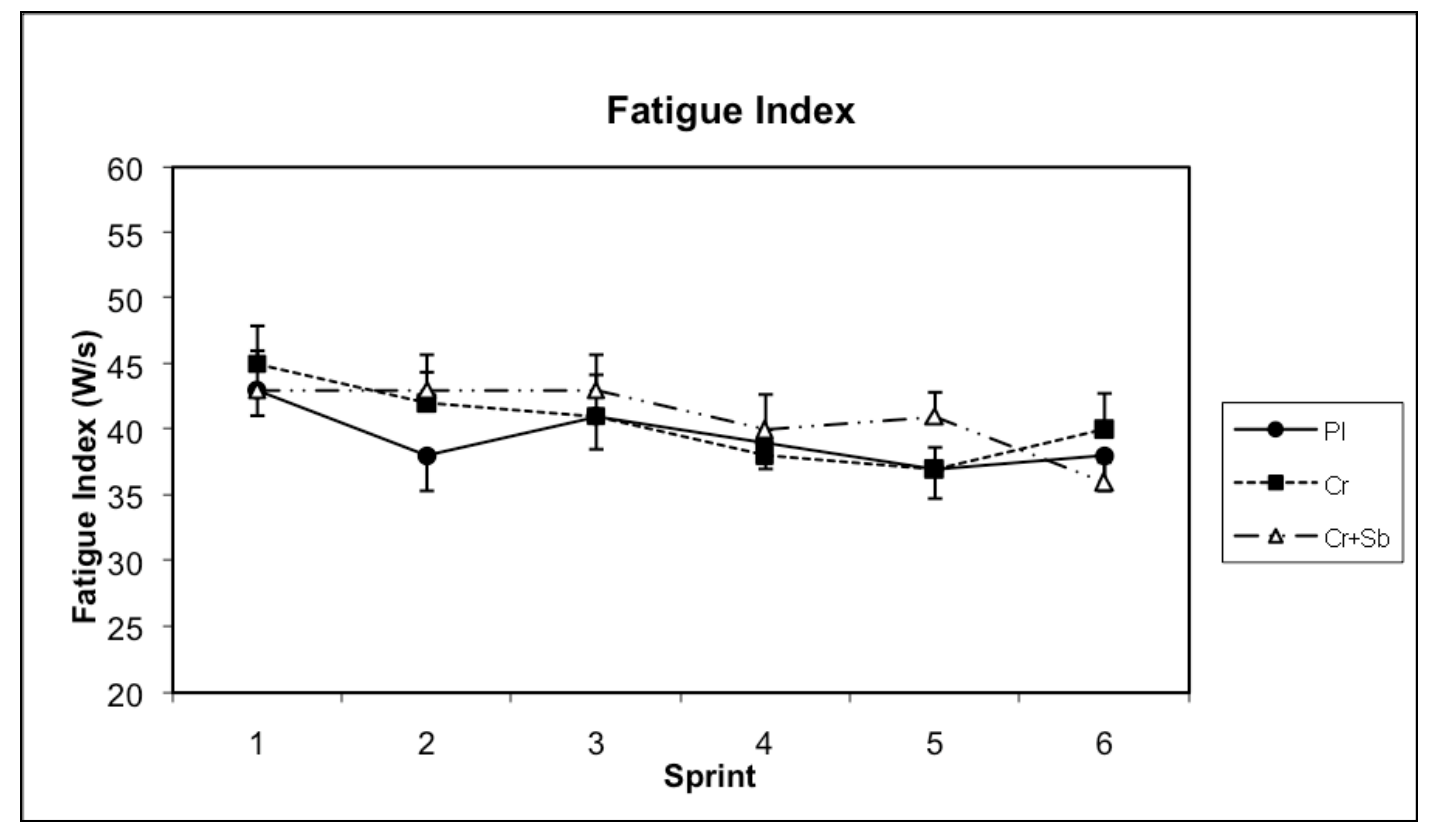

Figure 8. Fatigue Index values during the 6 repeated sprints in response to each condition. Values are in means $\pm \mathrm{SEM}$; W/s= Watts per second. $\mathrm{Pl}=$ placebo, $\mathrm{Cr}=$ Creatine alone, $\mathrm{CrSb}=$ creatine and sodium bicarbonate.

\section{Nutrition}

No main effect was found for condition when assessing the average caloric intake $(\mathrm{p}=$ 0.4739). The average energy intake of the 3 conditions consisted of the following percentage: (55 $\pm 1 \%$ for carbohydrate, $16 \% \pm 1 \%$ protein, and $29 \pm 1 \%$. 


\begin{tabular}{|c|c|c|c|c|}
\hline Variable & Sprint & Pl & $\mathrm{Cr}$ & $\mathrm{Cr}+\mathrm{Sb}$ \\
\hline \multirow[t]{6}{*}{ Peak Power (W) } & 1 & $1026 \pm 130$ & $1049 \pm 145$ & $1038 \pm 147$ \\
\hline & 2 & $944 \pm 106$ & $1024 \pm 126$ & $1022 \pm 143$ \\
\hline & 3 & $948 \pm 97$ & $969 \pm 142$ & $1005 \pm 116$ \\
\hline & 4 & $907 \pm 86^{*}$ & $925 \pm 87^{*}$ & $948 \pm 126^{*}$ \\
\hline & 5 & $884 \pm 102 *$ & $908 \pm 100 *$ & $944 \pm 113^{*}$ \\
\hline & 6 & $883 \pm 116^{*}$ & $924 \pm 118^{*}$ & $895 \pm 86^{*}$ \\
\hline \multirow[t]{6}{*}{ RPP (W/kg) } & 1 & $14.0 \pm 0.9$ & $14.3 \pm 1.0$ & $14.1 \pm 1.1$ \\
\hline & 2 & $12.9 \pm 1.2$ & $14.0 \pm 0.9$ & $13.9 \pm 0.9$ \\
\hline & 3 & $12.9 \pm 0.9$ & $13.2 \pm 1.0$ & $13.7 \pm 0.9$ \\
\hline & 4 & $12.4 \pm 1.3^{*}$ & $12.7 \pm 1.1^{*}$ & $13.0 \pm 1.2$ \\
\hline & 5 & $12.0 \pm 1.1^{*}$ & $12.4 \pm 1.0 *$ & $13.0 \pm 1.1 *$ \\
\hline & 6 & $11.9 \pm 1.3^{*}$ & $12.6 \pm 1.5^{*}$ & $12.7 \pm 0.8$ \\
\hline \multirow[t]{6}{*}{ Mean Power (W) } & 1 & $716 \pm 73$ & $724 \pm 69$ & $731 \pm 72$ \\
\hline & 2 & $686 \pm 56$ & $700 \pm 60$ & $712 \pm 72$ \\
\hline & 3 & $622 \pm 56^{*}$ & $670 \pm 56^{*}$ & $684 \pm 66^{*}$ \\
\hline & 4 & $641 \pm 54^{*}$ & $656 \pm 53^{*}$ & $668 \pm 63^{*}$ \\
\hline & 5 & $629 \pm 52 *$ & $646 \pm 67^{*}$ & $649 \pm 69^{*}$ \\
\hline & 6 & $623 \pm 56^{*}$ & $639 \pm 59^{*}$ & $651 \pm 62 *$ \\
\hline \multirow[t]{6}{*}{ RMP (W/kg) } & 1 & $9.8 \pm 0.5$ & $9.9 \pm 0.5$ & $9.8 \pm 0.4$ \\
\hline & 2 & $9.4 \pm 0.6$ & $9.6 \pm 0.6$ & $9.5 \pm 0.4$ \\
\hline & 3 & $9.0 \pm 0.7^{*}$ & $9.1 \pm 0.6^{*}$ & $9.2 \pm 0.6^{*}$ \\
\hline & 4 & $8.7 \pm 0.7^{*}$ & $9.0 \pm 0.7^{*}$ & $9.0 \pm 0.6^{*}$ \\
\hline & 5 & $8.6 \pm 0.8^{*}$ & $8.7 \pm 0.9^{*}$ & $8.7 \pm 0.7 *$ \\
\hline & 6 & $9.5 \pm 0.8^{*}$ & $8.7 \pm 0.8^{*}$ & $8.8 \pm 0.8^{*}$ \\
\hline \multirow[t]{6}{*}{ FI (W/kg) } & 1 & $43.5+6.9$ & $45.0 \pm 10.5$ & $43.0 \pm 10.9$ \\
\hline & 2 & $38.2 \pm 9.5$ & $45.2 \pm 8.6$ & $43.1 \pm 9.8$ \\
\hline & 3 & $41.2 \pm 8.9$ & $41.2 \pm 11.5$ & $43.7 \pm 9.8$ \\
\hline & 4 & $39.0 \pm 7.1$ & $38.5 \pm 7.3$ & $40.0 \pm 9.7$ \\
\hline & 5 & $37.5 \pm 8.0$ & $37.6 \pm 6.0$ & $41.7 \pm 6.7$ \\
\hline & 6 & $38.9 \pm 9.5$ & $40.2 \pm 10.0$ & $36.9 \pm 5.5$ \\
\hline
\end{tabular}

Table 4. Wingate sprint results for each sprint within each condition. Values are in means ( \pm $\mathrm{SD}) ; \mathrm{W}=$ Watts, $\mathrm{W} / \mathrm{s}=$ Watts per second, $\mathrm{W} / \mathrm{kg}=$ Watts per kilogram body mass. $\mathrm{Pl}=$ placebo, $\mathrm{Cr}=$ Creatine, $\mathrm{CrSb}=$ creatine and sodium bicarbonate. $*$ Significantly lower than sprint \#1. 


\section{Blood Analysis}

\section{Blood Bicarbonate}

For blood bicarbonate, a significant main effect was observed $(\mathrm{p}<0.0001)$ as well as a time effect $(\mathrm{p}<0.0001)$. Blood bicarbonate levels averaged across pre and post-test in the $\mathrm{CrSb}$ condition were significantly higher than both the creatine alone $(\mathrm{p}=<0.0001)$ and placebo $(\mathrm{p}<$ 0.0001) conditions. A significant condition $x$ time interaction was also observed $(p=0.0004)$. Post hoc testing indicated significantly higher pre-test bicarbonate concentrations in the combined supplementation condition $(\mathrm{CrSb})$ compared to creatine alone $(10.2 \%, \mathrm{p}=<0.001)$ and placebo $(11.6 \%, \mathrm{p}=0.001)$. Post-exercise bicarbonate concentrations in each condition were not significantly different in either group. In all conditions, pre-test blood $\mathrm{pH}$ levels were significantly higher than post-test.

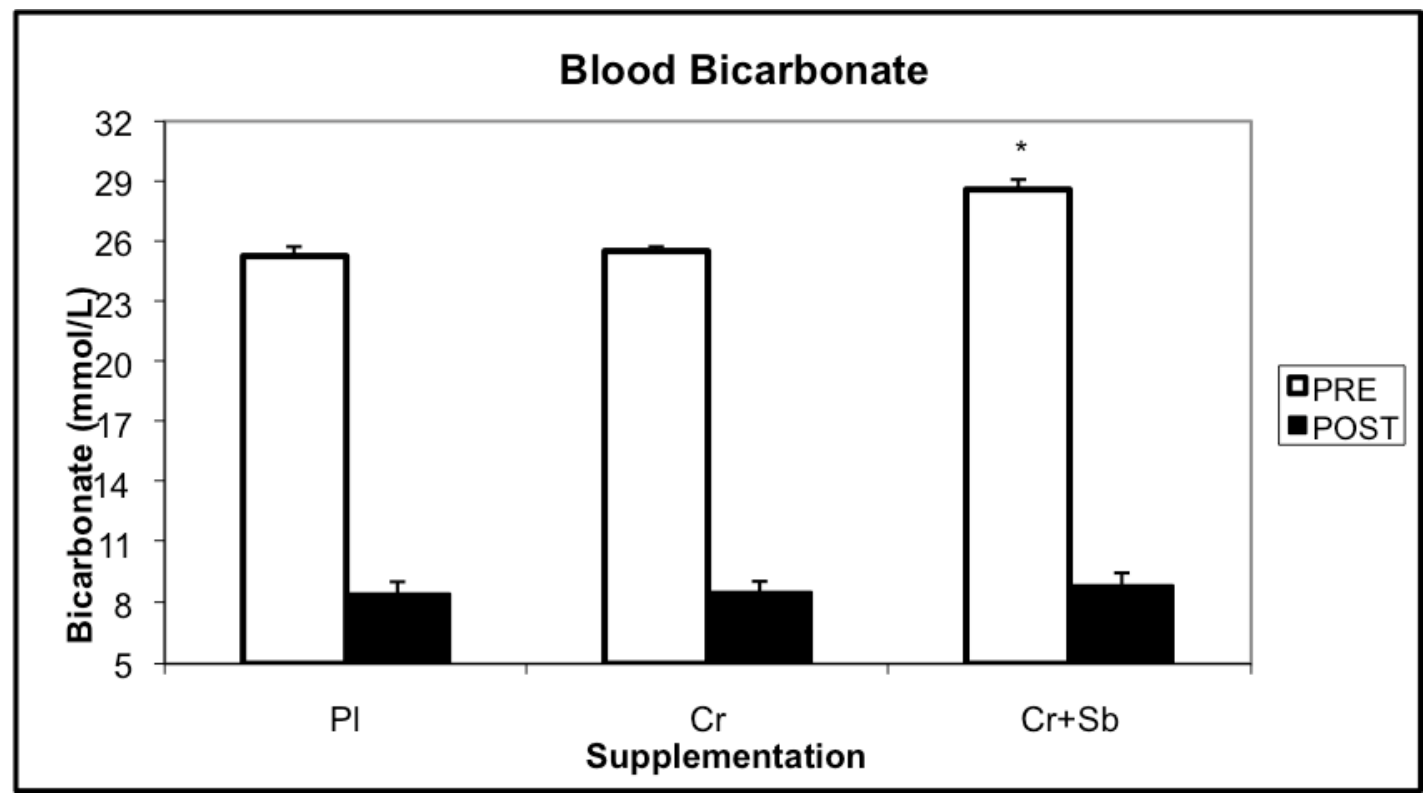

Figure 9. Pre and post Wingate blood bicarbonate levels of the three conditions. $\mathrm{Pl}=$ placebo, $\mathrm{Cr}$ $=$ Creatine alone, $\mathrm{CrSb}=$ creatine and sodium bicarbonate. Values are in means $\pm \mathrm{SE}$. * Denotes significantly higher than placebo and creatine alone. 


\section{Blood pH}

For blood $\mathrm{pH}$, a significant main effect was observed for condition $(\mathrm{p}=0.0406)$.

However, a Tukey's post hoc analysis showed that there was no difference within the groups. A trend towards a higher pre-test $\mathrm{pH}$ in the combined creatine and sodium bicarbonate condition when compared to creatine $(p=0.071)$ and placebo $(p=0.074)$ was observed. There was a time effect $(\mathrm{p}<0.0001)$. In all conditions, pre-test blood $\mathrm{pH}$ levels were significantly higher than post-test. No significant condition $\mathrm{x}$ time interaction was observed $(\mathrm{p}=0.4874)$.

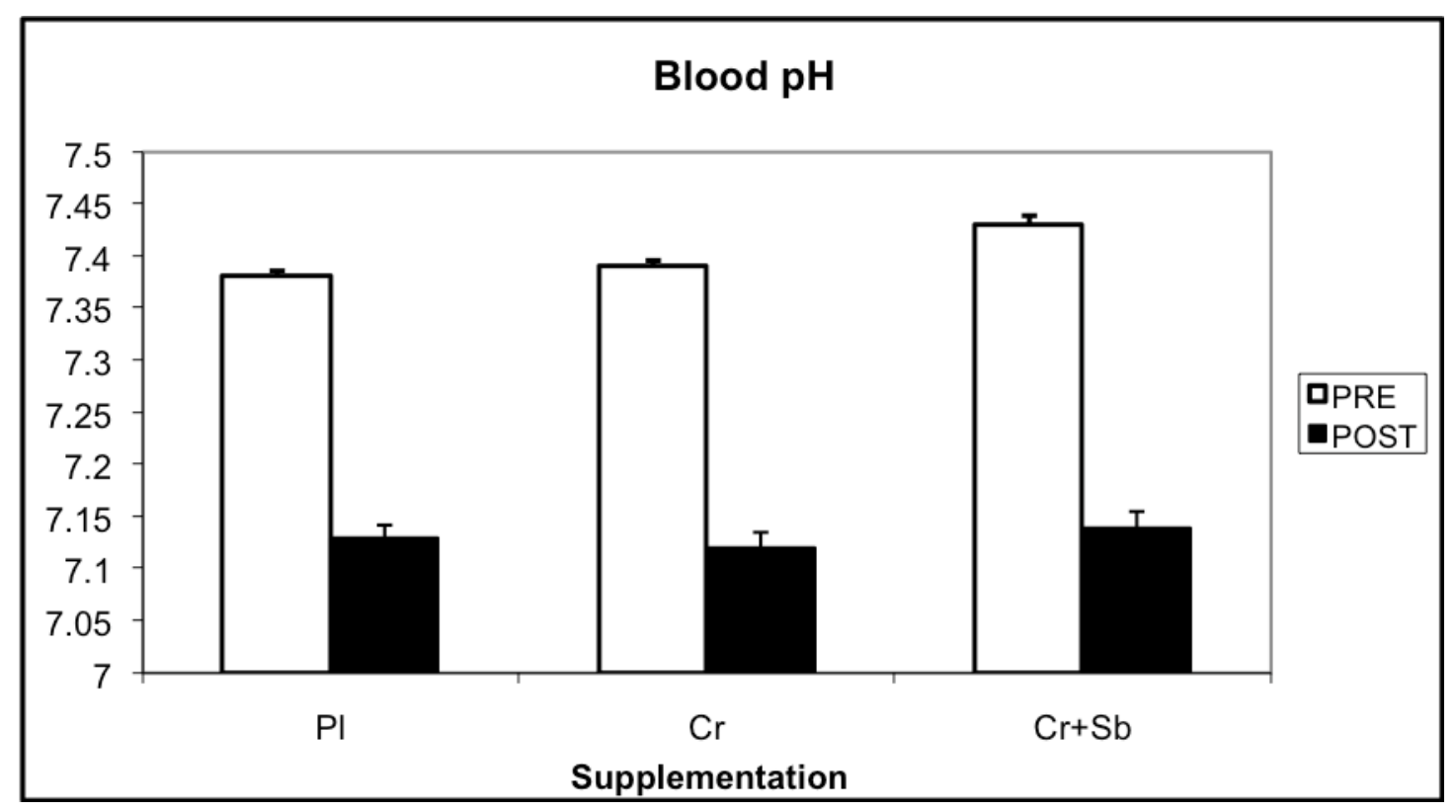

Figure 10. Pre and post Wingate blood $\mathrm{pH}$ levels of the three conditions. $\mathrm{Pl}=$ placebo, $\mathrm{Cr}=$ Creatine alone, $\mathrm{CrSb}=$ creatine and sodium bicarbonate. Values are in means $\pm \mathrm{SE}$.

\section{Blood Lactate}

For blood lactate, no main effect was observed for condition $(\mathrm{p}=0.2512)$. There was a time effect $(\mathrm{p}<0.0001)$ which indicated that across each condition, pre-test lactate levels were significantly lower than post-test levels. No significant condition $\mathrm{x}$ time interaction was observed $(\mathrm{p}=0.5173)$. Post hoc testing indicated no significant differences in blood lactate levels before 
or after the sprint bouts.

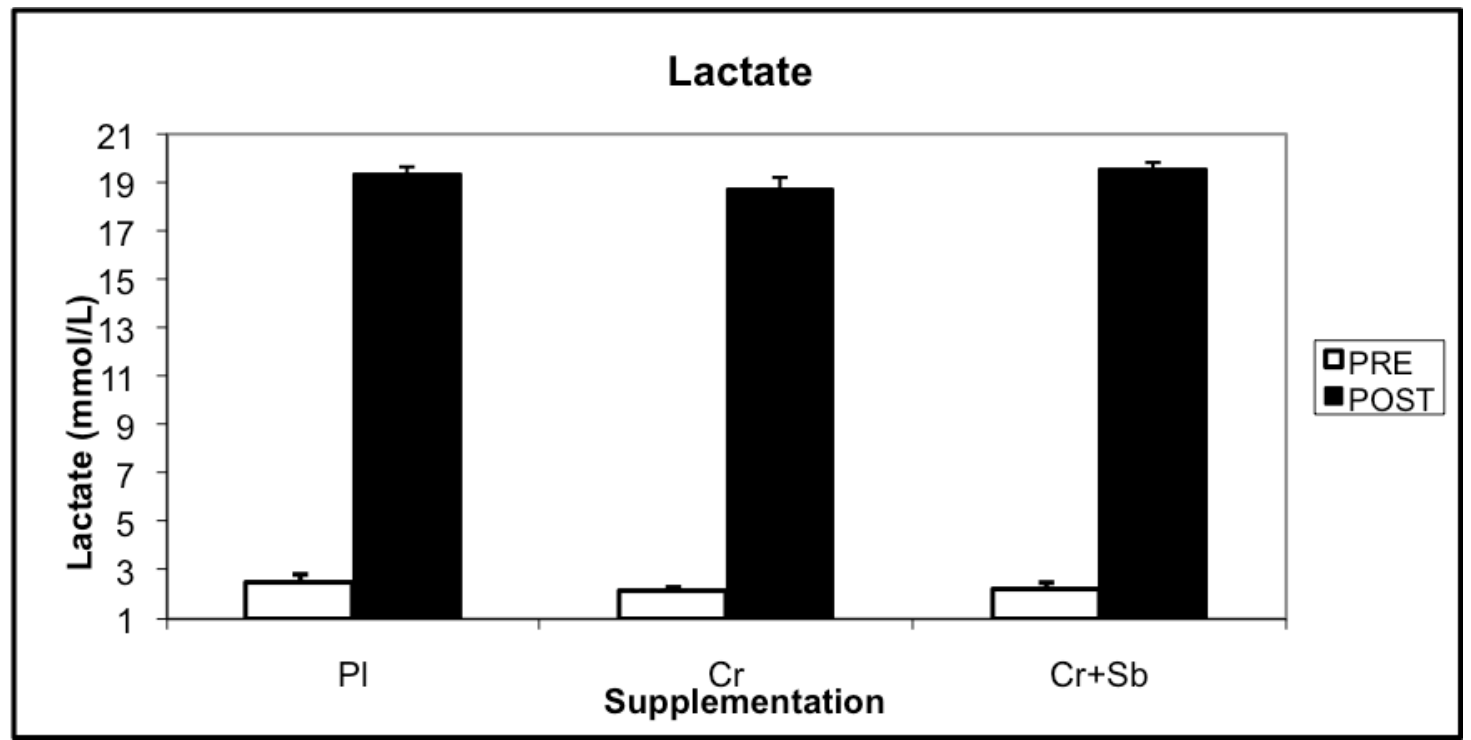

Figure 11. Pre and post Wingate blood $\mathrm{pH}$ levels of the three conditions. $\mathrm{Pl}=$ placebo, $\mathrm{Cr}=$ Creatine alone, $\mathrm{CrSb}=$ creatine and sodium bicarbonate. Values are in means $\pm \mathrm{SE}$.

\section{Perception of Fatigue and Gastrointestinal Distress}

For this study, fatigue and gastrointestinal distress questionnaires were evaluated after each sprint trial in each condition. Results in their mean values $( \pm \mathrm{SD})$ are presented in Table 5 . For perceptions of fatigue, no main effect was observed $(p=0.6080)$ between the conditions. A time effect was observed for perceptions of fatigue $(p<0.001)$. In each of the conditions, perceptions of fatigue in sprints $3,4,5$ and 6 were significantly higher than in sprints 1 and 2 . No significant interaction of condition $\mathrm{x}$ sprint was found.

Gastrointestinal distress and systemic discomfort was assessed in 3 sections: upper abdominal, lower abdominal, and systemic areas. For each of the conditions, no main effect for condition was observed. $(\mathrm{p}=0.0672, \mathrm{p}=0.7349, \mathrm{p}=0.6706)$. A significant time effect was observed for each of the 3 sections $(p<0.001)$. 


\begin{tabular}{|c|c|c|c|c|}
\hline Variable & Sprint & Pl & $\mathrm{Cr}$ & $\mathrm{Cr}+\mathrm{Sb}$ \\
\hline \multirow[t]{6}{*}{ Fatigue } & 1 & $1.9 \pm 0.9$ & $2.6 \pm 1.7$ & $1.8 \pm 0.6$ \\
\hline & 2 & $2.7 \pm 1.0$ & $3.4 \pm 1.9$ & $3.0 \pm 1.1$ \\
\hline & 3 & $4.3 \pm 1.0$ & $4.6 \pm 2.2$ & $4.3 \pm 0.6$ \\
\hline & 4 & $5.2 \pm 1.0$ & $5.8 \pm 1.9$ & $5.6 \pm 1.0$ \\
\hline & 5 & $6.7 \pm 1.1$ & $7.0 \pm 1.8$ & $6.8 \pm 1.2$ \\
\hline & 6 & $7.9 \pm 1.2$ & $7.9 \pm 1.6$ & $8.0 \pm 1.3$ \\
\hline \multirow[t]{6}{*}{ Upper Abdominal } & 1 & $1.0 \pm 0.0$ & $1.1 \pm 0.2$ & $1.1 \pm 0.3$ \\
\hline & 2 & $1.1 \pm 0.2$ & $1.3 \pm 0.6$ & $1.6 \pm 1.0$ \\
\hline & 3 & $1.5 \pm 0.9$ & $1.5 \pm 0.8$ & $1.0 \pm 0.9$ \\
\hline & 4 & $1.7 \pm 1.3$ & $2.3 \pm 2.0$ & $2.0 \pm 1.2$ \\
\hline & 5 & $1.7 \pm 1.5$ & $2.6 \pm 2.7$ & $2.4 \pm 1.6$ \\
\hline & 6 & $2.2 \pm 1.9$ & $3.1 \pm 2.9$ & $2.8 \pm 2.1$ \\
\hline \multirow[t]{6}{*}{ Lower Abdominal } & 1 & $1.1 \pm 0.2$ & $1.1 \pm 0.2$ & $1.2 \pm 0.5$ \\
\hline & 2 & $1.2 \pm 0.5$ & $1.3 \pm 0.6$ & $1.6 \pm 0.9$ \\
\hline & 3 & $1.4 \pm 1.3$ & $1.6 \pm 1.1$ & $1.7 \pm 0.9$ \\
\hline & 4 & $1.8 \pm 1.5$ & $2.0 \pm 1.8$ & $2.0 \pm 1.6$ \\
\hline & 5 & $1.8 \pm 1.8$ & $2.5 \pm 2.6$ & $2.2 \pm 1.7$ \\
\hline & 6 & $2.5 \pm 2.4$ & $3.0 \pm 2.9$ & $2.6 \pm 2.0$ \\
\hline \multirow[t]{6}{*}{ Systemic } & 1 & $0.9 \pm 0.0$ & $1.3 \pm 0.8$ & $1.1 \pm 0.3$ \\
\hline & 2 & $1.2 \pm 0.4$ & $1.8 \pm 1.1$ & $1.7 \pm 0.2$ \\
\hline & 3 & $2.0 \pm 1.0$ & $2.7 \pm 1.7$ & $2.4 \pm 1.3$ \\
\hline & 4 & $2.8 \pm 1.4$ & $3.4 \pm 2.2$ & $3.4 \pm 2.1$ \\
\hline & 5 & $3.8 \pm 2.3$ & $4.3 \pm 2.7$ & $3.9 \pm 2.4$ \\
\hline & 6 & $4.9 \pm 2.1$ & $5.1 \pm 3.0$ & $4.8 \pm 2.6$ \\
\hline
\end{tabular}

Table 5. Perceptions of fatigue and GI discomfort results during the repeated Wingate test in response to each condition. Values are in means $( \pm \mathrm{SD}) . \mathrm{Pl}=$ Placebo, $\mathrm{Cr}=$ Creatine, $\mathrm{Cr}+\mathrm{Sb}=$ Creatine and Sodium Bicarbonate. Each of the responses are a rating from (0-10). 


\section{Chapter 5}

\section{$\underline{\text { Discussion }}$}

The goal of this study was to examine whether the addition of sodium bicarbonate to creatine supplementation would further improve markers of exercise performance and fatigue compared to creatine supplementation alone. The main findings were: 1) the addition of sodium bicarbonate to creatine produced greater relative peak power, peak power, and mean power values than placebo; 2) combining creatine and sodium bicarbonate demonstrated the greatest attenuation of decline in relative peak power over 6 repeated sprints; and 3) two days of creatine supplementation was sufficient to increase repeated sprint performance compared to placebo. However, in contrast to our hypotheses, no benefits from either supplementation were observed for relative mean power, fatigue index, and perception of fatigue.

A novel finding in the current study is an apparent difference in exercise performance when adding sodium bicarbonate to creating supplementation. In this study, the $\mathrm{Cr}+\mathrm{Sb}$ supplementation resulted in approximately $7 \%$ greater relative peak power than the placebo condition during the repeated Wingate sprints. Although relative peak power values were not significantly different from the $\mathrm{Cr}$ supplementation, the $\mathrm{Cr}+\mathrm{Sb}$ supplementation demonstrated the greatest attenuation of decline in relative peak power values over the 6 repeated sprints. Furthermore, $\mathrm{Cr}+\mathrm{Sb}$ also produced $4.5 \%$ greater peak power and 3.5\% greater mean power values than placebo. In the one other study evaluating the combined impact of the two supplements, Mero et al. (2004) found that combining creatine and sodium bicarbonate improves interval swim performance. However, a potential confound in that study is that the supplementation was not compared to a creatine alone supplementation. Therefore, it is unclear how large the single role of creatine or sodium bicarbonate played in the performance benefit. 
To our knowledge, this current study is the first to directly compare the effects of a combined creatine and sodium bicarbonate supplementation to that of creatine alone. Our results are consistent with and extend the findings of Mero et al. (2004), showing that adding sodium bicarbonate to creatine further improves relative peak power, peak power, and mean power during repeated sprints. Because of this, we now have more insight in their combined performance effect.

We hypothesized that since both creatine and sodium bicarbonate work in independent pathways, have no interactive affects, and that they both have similar effects in improving repeated sprints, there would be an additive effect on exercise performance when compared to $\mathrm{Cr}$ alone. When examining the mechanism of creatine and sodium bicarbonate independently, research suggests that they both aid in repeated sprint performance, primarily through increasing creatine and bicarbonate stores. In creatine's mechanism of action, increased PCr availability allows for a more rapid resynthesis of ATP (24). Furthermore, increased PCr is thought to aid in the shuttling of high-energy phosphates from the site of muscular contraction to the mitochondrial membrane where ATP is produced. Specifically, this process of rapid resynthesis of ATP and PCr appears to be enhanced during recovery periods of high-intensity intermittent exercise (10). This may be attributed to the rate of flux through the creatine kinase reaction at the mitochondrial membrane during rest periods (10).

During sodium bicarbonate supplementation, it is understood that by tapping the bicarbonate stores in the blood, our buffering capacity is enhanced. During high intensity exercise, accumulation of hydrogen ions in the muscle result in decreased blood and muscle $\mathrm{pH}$ (45). Research suggests that this decrease in muscular $\mathrm{pH}$ resulting from accumulation of hydrogen ions $(\mathrm{H}+)$ hinders aspects of performance such as power output and fatigue by a 
disruption of contractility within the myofilaments in muscle $(32,52)$. It is thought that by increasing our bicarbonate stores from sodium bicarbonate supplementation, this would allow for a greater efflux of hydrogen ions out of the muscle cell therefore decreasing fatigue and power output declines. In support, we found that $\mathrm{Cr}+\mathrm{Sb}$ condition resulted in a greater attenuation of the decline in power output when compared to both the $\mathrm{Cr}$ and $\mathrm{Pl}$ condition (Table 3). Even though the exact mechanism of action for remains unclear for creatine and sodium bicarbonate, both have been shown to increase markers of performance $(1,3,4,7-9,11-12,16,31,34,37-41$, $43-45,47,51,53,55,65,68)$. Here, it may be true that adding sodium bicarbonate to creatine supplementation may augment a greater buffering capacity than creatine alone; therefore, resulting in greater power output and preventing power output declines during repeated sprints. Furthermore, when addressing the G.I. discomfort across each condition, there were no differences between the groups. This suggests that the administration of two-day combined supplementation of creatine and sodium bicarbonate may yield performance benefits without significant side-effects.

\section{Blood Measurements}

Previous research has shown that ingesting sodium bicarbonate in 0.5 grams $/ \mathrm{kg}$ per day of creatine raises blood bicarbonate concentrations (43). This current study observed similar increases in blood bicarbonate concentrations resulting from the same $0.5 \mathrm{~g} / \mathrm{kg}$ dose for 2 days of sodium bicarbonate. When subjects ingested the $\mathrm{Cr}+\mathrm{Sb}$ supplementation, resting blood bicarbonate levels were $11.6 \%$ higher than the $\mathrm{Pl}$ supplementation and $10.2 \%$ higher than $\mathrm{Cr}$ supplementation. In theory, the higher levels of bicarbonate in the blood from the $\mathrm{Cr}+\mathrm{Sb}$ supplementation would yield higher resting blood $\mathrm{pH}$ levels, therefore triggering a greater efflux of hydrogen ion removal out of the muscle cell (51). In this study, the greater relative peak 
power, peak power, mean power, along with the greater attenuation of decline in power output found in the $\mathrm{Cr}+\mathrm{Sb}$ supplementation may be due to the increased resting blood bicarbonate levels. This, in theory, would allow for the greater efflux of hydrogen ions out of the muscle cell and, therefore, allow for greater work and intensity to be performed. These results must be taken with caution, because although resting bicarbonate levels were significantly higher in the $\mathrm{Cr}+\mathrm{Sb}$ supplementation, this pattern did not hold true for blood $\mathrm{pH}$. Although no statistical differences were found in resting blood $\mathrm{pH}$ values, there was a trend towards a higher overall blood $\mathrm{pH}$ value in the $\mathrm{Cr}+\mathrm{Sb}$ supplementation when compared to $\mathrm{Cr}$ and $\mathrm{Pl}(\mathrm{p}=0.074, \mathrm{p}=0.071)$. This may be due to the fact that blood was only sampled at pre and post Wingate sprints. Future research should assess $\mathrm{pH}$ and bicarbonate concentrations more frequently during repeated sprints to determine the time course of each measurement and whether they are related to increased performance.

In addition to the positive results in the combined supplementation of creatine and sodium bicarbonate, we found that only two days of creatine supplementation, without a loading phase, was sufficient to increase power output compared to placebo. A traditional loading dose for creatine supplementation is 20-25grams for 4-7 days (61). The landmark findings by Harris et al. (1992) and Greenhaff (1994) stating that this dose increases total creatine stores by up to $25 \%$, made way for much research to be conducted on creatine loading for several days. However, little research has been conducted on the benefits of a strict 2-day dosing period. This may be because many athletes who supplement on creatine strive for the long-term physiological adaptations of creatine such as weight gain and increasing muscle size. In this study, relative peak power values for the creatine supplementation condition were $5.8 \%$ greater than the values of the placebo. This adds to the already well-established findings that creatine supplementation 
improves performance. More specifically, this study found that only 2-days of loading would be sufficient to see performance benefits. These results extend the findings in Ziegenfuss et al. (2002), who found that a shorter 3-day creatine supplementation resulted in significantly higher relative peak power values than placebo in same 610 -second Wingate sprint protocol as this current study. Furthermore, initial research on creatine by Harris et. al (1992) observed that peak creatine uptake in the muscle after a $25 \mathrm{~g} /$ day loading dose occurred after 2 days of supplementation. Vandenberge et al. (1999) also reported that 2 days of creatine loading of 25 grams significantly elevated PCr stores by $11 \%$. Although blood and muscle creatine samples were not administered in this study, the higher relative peak power values during the creatine supplementation suggests that 2 days of supplementation may be sufficient in increasing $\mathrm{PCr}$ stores resulting in the greater power output results observed. The real-world impact of these results is that an 2-day supplementation may be a more convenient dose for athletes interested in acute benefits of creatine without having to load for longer periods of time.

On the contrary, several studies have failed to find an acute performance benefit from creatine supplementation. Eckerson et al. (2005) failed to find a significant improvement on anaerobic working capacity in males and females after creatine supplementation. Similarly, Ahmun et al. (2005) found that creatine supplementation failed to demonstrate a positive effect in power output and fatigue index in rugby players. This may be due to methodological issues or the fact that $25 \%$ of people who supplement on creatine are "non-responders" (24).

Although the results from this study provide positive results for the addition of sodium bicarbonate to creatine, this study had several limitations. Even though all 13 subjects underwent the repeated sprint analysis, due to cost, blood samples were assessed in only 10 subjects. Because of this, it remains unclear what would have happened to $\mathrm{pH}$ and bicarbonate 
concentrations during the repeated sprints. In addition, blood creatine and PCr levels were not examined. Without these measurements, it is unknown if creatine levels in the blood were elevated during the supplementation period. Second, this study was over the course of 9-weeks because of $3 \times 3$ washout periods. Because of this long testing period, there may be variability in the results due to the specific condition of the subjects during each testing period. Specifically, 2 subjects during the first and second week of testing became sick. Subjects informed the test administrator that they had felt cold-like symptoms. However, we saw no statistical carry-over effect of treatments, suggesting that variability was minimal. Third, subjects were not screened on sport/exercise specificity. Several, but not all, subjects were recreational triathletes and others were anaerobic athletes. Subjects were assessed on VO2 max to determine if they were sufficiently fit for the study. Anaerobic athletes tend to show greater power output values and greater fatigue, whereas, the opposite holds true for aerobic athletes. Because of these differences were not accounted for, it is unknown whether the performance benefits are more apparent in anaerobic or aerobic athletes. However, a benefit to this study was that it was completed singlesexed. This eliminated any physiological differences that may have affected performance. Although research suggests that women yield benefits from creatine and sodium bicarbonate supplementation alone (68), it is unknown what the effects of the combination of creatine and sodium bicarbonate are in women. Lastly, this study did not have a sodium bicarbonate alone supplementation. With this, there is no way of knowing if sodium bicarbonate alone would produce enhanced results when compared to creatine alone and combined creatine and sodium bicarbonate.

In summary, we found that, 1) the addition of sodium bicarbonate to creatine supplementation produced greater relative peak power, peak power, and mean power values than 
placebo, 2) combining creatine and sodium bicarbonate demonstrated the greatest attenuation of decline in relative peak power over 6 repeated sprints and 3) the administration of a 2-day dose of creatine is sufficient to improve relative peak power values in 6 repeated sprints. Considering that this current study as well as research conducted by Mero et. al (2004) found benefits from combining these two well-known ergogenic aids, this gives positive rational to continue to research these two supplements in combination. More must be concluded before we can endorse using a combined supplementation of creatine and sodium bicarbonate for performance enhancement. Future research on a greater sample size, a specific athletic population, various exercise modes, and comparing results with a sodium bicarbonate alone supplementation would be beneficial in determining if this combined supplementation is worthwhile. Furthermore, because there is an abundance of research on the effects of both creatine and sodium bicarbonate alone, more research needs to be conducted to determine the physiological mechanism of their combined effect.

\section{Practical Applications}

Our data suggests that a two-day supplementation of creatine and sodium bicarbonate may increase relative peak power output by $7 \%$ compared to placebo. Furthermore, this study found that a combined supplementation of creatine and sodium bicarbonate delays fatigue greater than creatine alone. Although these increases in power output and decreases in fatigue may seem minimal, at the elite level, this can have a profound effect on improving high intensity performance. At the elite level in athletics, the difference between first and second place in short, high intensity sports may be minuscule. An 7\% increase in power output combined with a lower fatigue resulting from a combined supplementation of creatine and sodium bicarbonate may give an athlete a competitive advantage over non-supplemented athletes. Furthermore, when 
considering that there were no significant gastrointestinal side-effects found from each of these conditions, this suggests that the power output benefits gained from the combined supplementation of creatine and sodium bicarbonate are worthwhile. 


\section{References}

1. Aaserud R, Gramvik P, Olsen S, Jensen J. Creatine supplementation delays onset of fatigue during repeated bouts of sprint running. Scandinavian Journal of Medicine and Science in Sports 8: 247-251, 1998.

2. Ahmun R, Tong R, Grimshaw P. The effects of acute creatine supplementation on multiple sprint cycling and running performance in rugby players. Journal of Strength and Conditioning Research 19: 92-97, 2005.

3. Balsom P, Ekblom B, Soderlund K, Sjodin B, Hultman E. Creatine supplementation and dynamic high-intensity intermittent exercise. Scandinavian Journal of Medicine and Science in Sports and Exercise 3: 143-149 1993.

4. Balsom P \& Sjodin B. Skeletal muscles metabolism during short duration high intensity exercise. Acta Physiologica Scandinavica, 154: 303-310 1995.

5. Bemben $\mathrm{M} \&$ Lamont H. Creatine supplementation and exercise performance: recent findings. Sports Medicine: 35(2), 107-125, 2005.

6. Birch R, Noble D, Greenhaff P. The influence of dietary creatine supplementation on performance during repeated abouts of maximal isokinetic cycling in man. European Journal of Applied Physiology and Occupational Physiology 69 (3) 268-270, 1994.

7. Bishop D \& Claudius. Effects of induced metabolic alkalosis on prolonged intermittent-sprint performance. Medicine and Science in Sports and Exercise 37 (5): 759-767, 2005.

8. Bishop D, Edge J, Davis C, Goodman C. Induced metabolic alkalosis affects muscle metabolism and repeated-sprint ability. Medicine and Science in Sports and Exercise 36 (5) 807-813, 2004.

9. Burke LM \& Pyne. Bicarbonate loading to enhance training and competitive performance. International Journal of Sports Physiology and Performance (2):93-97, 2007.

10. Casey A, \& Greenhaff P. Does dietary creatine supplementation play a role in skeletal muscle metabolism and performance? American Journal of Clinical Nutrition: 72, 7-17, 2000.

11. Costill D, Verstappen F, Kuipers H, Janssen E, Fink W. Acid-base balance during repeated bouts of exercise: influence of HCO3. International Journal of Sports Medicine: 5(5), 228-231, 1984. 
12. Dawson B, Cutler M, Moody A, Lawrence S, Goodman C, Randall N. Effects of oral creatine loading on single and repeated maximal short sprints. The Australian Journal of Science and Medicine in Sports 27 (3): 56-61, 1995.

13. Delecluse C, Diels R, Goris M. Effect of creatine supplementation on intermittent sprint running performance in highly trained athletes. Journal of Strength and Conditioning Research 17 (3): 446-454, 2003.

14. Demant TW \& Rhodes EC. Effects of creatine supplementation of exercise performance. Sports Medicine 28(1), 49-60, 1999.

15. Dotan R \& Bar-Or. Load Optimization for the wingate anaerobic test. European Journal of Applied Physiology 51: 409-417, 1983.

16. Douroudos II, Fatouros IG, Gourgoulis V, Jamurtas AZ, Tsitsios T, Hatzinikolaou A, Margonis K, Mavromatidis K, Taxildaris K. Dose-related effects of prolonged $\mathrm{NaHCO} 3$ ingestion during high-intensity exercise. Medicine and Science in Sports and Exercise 38(10): 1746-1753, 2006.

17. Earnest CP, Snell PG, Rodriguez R, Almada A, Mitchell T. The effect of creatine monohydrate ingestion on anaerobic power indices, muscularm strength and body composition. Acta Physiol Scand 153(2): 207-209, 1995.

18. Eckerson J, Stout J, Moore G, Stone N, Iwan K, Gebauer A, Ginsberg R. Effect of creatine phosphate supplementation on anaerobic working capacity and body weight after two and six days of loading in men and women. Journal of Strength and Conditioning Research 19 (4): 756-763, 2005.

19. Englehardt M, Neumann G, Berbalk A, Reuter I. Creatine supplementation in endurance sports. Medicine and Science in Sports and Exercise (7) 1123-1129.

20. Gaitanos G, Nevill M, Books S, Williams C. Repeated bouts of sprint running after induced alkalosis. Journal of Sports Sciences: 9(4), 355-370.

21. Gill N, Hall R, Blazevich A. Creatine serum is not as effective as creatine powder for improving cycle sprint performance in competitive male team sports athletes. Journal of Strength and Conditioning Research 18 (2) 272-275.

22. Glaister M. Multiple sprint work: Methodological, physiological, and experimental issues. International Journal of Sports Physiology and Performance 3: 107-112, 2008.

23. Greenhaff P. Creatine and its application as an ergogenic aid. International Journal of Sports Nutrition 5: 100-110, 1995. 
24. Greenhaff P, Bodin K, Soderlund K, Hultman E. Effect of oral creatine supplementation on skeletal muscle phosphocreatine resynthesis. American Journal of Physiology 266: 725-730, 1994.

25. Greenhaff P, Casey A, Short A, Harris R, Soderlund K, Hultman E. Influence of oral creatine supplementation of muscle torque during repeated bouts of maximal voluntary exercise in man. Clinical Sciences 84: 565-571, 1993.

26. Harris R, Soderlund K, Hultman E. Elevation of creatine in resting and exercised muscle of normal subjects by creatine supplementation. Clinical Sciences 83: 367-374, 1992.

27. Havenetidis $\mathrm{K}$, Matsouka $\mathrm{O}$, Cooke $\mathrm{C}$, Theodorou $\mathrm{A}$. The use of varying creatine regimens on sprint cycling. Journal of Sports Science and Medicine (2), 88-97, 2003.

28. Hultman E, Soderland K, Timmons J, Cederblaad G, Greenhaff P. Muscle creatine loading in men. Journal of Applied Physiology (81), 232-237, 1996

29. Horswill C, Costill D, Fink W, Flynn M, Kirwan J, Mitchell J, Houmard J. Influence of sodium bicarbonate on sprint performance: relationship to dosage. Medicine and Science in Sports and Exercise: 20(6) 566-569, 1988.

30. Inbar O, Rotstein A, Jacobs I, Kaiser P, Dlin R, Dotan R. The effects of alkaline treatment on short-term maximal exercise. Journal of Sports Sciences: 1(2), 95-104, 1983

31. Izquierdo M, Ibanez J, Gonzalez-Baldillo J, Gorostiaga E. Effects of creatine supplementation on muscle power, endurance, and sprint performance. Medicine and Science in Sports and Exercise 34 (2): 332-343, 2002.

32. Lavender G \& Bird S. Effect of sodium bicarbonate ingestion upon repeated sprints. British Journal of Sports Medicine 23: 41-45, 1989.

33. Law Y, Ong W, GillianYap T, Lim S, Chia E. Effects of two and five days of creatine loading on muscular strength and anaerobic power in trained athletes. Journal of Strength and Conditioning Research 23 (3): 906-914.

34. Lindh AM, Peyrebrune MC, Ingham SA, Bailey DM, Folland JP. Sodium bicarbonate improves swimming performance. International Journal of Sports Medicine 29: 519-523, 2008.

35. Materko W, Santos E, Da Silva Novaes. Effect of bicarbonate supplementation on muscular strength. Journal of Exercise Physiology Online 11(6): 25-33, 2008.

36. McArdle W, Katch F, Katch V. Exercise Physiology: Energy, Nutrition, and Human Performance. Baltimore: Lippincott Williams \& Williams, 2007, p.568-603. 
37. McNaughton L. Effects of doseage on 60 second cycle ergometry. Journal of Sports Sciences. 10: 415-423, 1992.

38. McNaughton L. Sodium bicarbonate ingestion and its effect on anaerobic exercise of various durations. Journal of Sports Sciences 10: 425-435, 1992

39. McNaughton L, Backx K, Palmer G, Strange N. Effects of chronic bicarbonate ingestion on the performance of high intensity work. European Journal of Applied Physiology 80: 333-336, 1999.

40. McNaughton L \& Cedaro R. The effect of sodium bicarbonate on rowing ergometer performance in elite rowers. The Australian Journal of Science and Medicine in Sport 23 (3) 66-69, 1991.

41. McNaughton L, Dalton B, Palmer G. Sodium bicarbonate can be used as an ergogenic aid in high-intensity, competitive cycle ergometry of 1 hour duration. European Journal of Applied Physiology 80: 64-69, 1999.

42. McNaughton L, Dalton B, Tarr J. The effects of creatine supplementation on highintensity exercise performance in elite performers. European Journal of Applied Physiology and Occupational Physiology 78 (3) 236-240, 1998.

43. McNaughton L \& Thompson D. Acute versus chronic sodium bicarbonate ingestion and anaerobic work and power output. Journal of Sports Medicine and Fitness 41 (4): 456$462,2001$.

44. McNaughton L, Siegler J, Midgley A. Ergogenic effects of sodium bicarbonate. Current Sports Medicine Reports 7 (4): 230-236, 2008.

45. Mero A, Keskinen K, Malvela M, Sallinen J. Combined creatine and sodium bicarbonate supplementation enhances interval swimming. Journal of Strength and Conditioning Research 18 (2): 306-310, 2004.

46. Petroczi A \& Naughton D. Supplement use in sport; is there a potentially dangerous incongruence between rationale and practice? Journal of Occupational Medicine and Toxicology 2(4), 1-6, 2007.

47. Peyrebrune M, Nevill M, Donaldson F, Cosford D. The effects of oral creatine supplementation on performance in single and repeated sprint swimming. Journal of Sports Sciences 16: 271-279, 1998.

48. Pfeiffer B, Cotterill A, Grathwohl D, Stellingwerff T, Jeukendrup A. The effect of carbohydrate gels on gastrointestinal tolerance during a 16-km run. International Journal of Sport Nutrition and Exercise Metabolism 19: 485-503, 2009. 
49. Portington K, Pascoe D, Webster M, Anderson L, Rutland R, Gladden B. Effect of induced alkalosis on exhaustive leg press performance. Medicine and Science in Sports and Exercise: 30(4), 523-528, 1998.

50. Preen D, Dawson B, Goodman C, Lawrence S, Beilby J, Ching S. Effect of creatine loading long-term sprint exercise performance and metabolism. Medicine and Science in Sports and Exercise 33(5): 814-821, 2001

51. Requena B, Zabala M, Padial P, Feriche B. Sodium bicarbonate and sodium citrate: ergogenic aids? Journal of Strength and Conditioning Research. 19(1), 2005.

52. Robergs R, Hutchinson K, Hendee S, Madden S, Siegler J. Influence of pre-exercise acidosis and alkalosis on the kinetics of acid-base recovery following intense exercise. International Journal of Sports Nutrition and Exercise Metabolism 14: 1-16, 2005.

53. Siegler JC, Keatley S, Midgley AW, Nevill AM, McNaughton LR. Pre-exercise alkalosis and acid-base recovery. International Journal of Sports Medicine 28: 1-7, 2007.

54. Silver MD. Use of ergogenic aids by athletes. Journal of American Academy of Orthopaedic Surgeons 9(1), 61-70, 2001.

55. Skare O, Skadberg O, Wisnes A. Creatine supplementation improves sprint performance in male sprinters. Scandinavian Journal of Medicine and Science in Sports 11: 96-102, 2001.

56. Spencer M, Bishop D, Dawson B, Goodman C, Duffield R. Metabolism and performance in repeated cycle sprints: active versus passive recovery. Medicine and Science in Sports and Exercise 38 (8): 1492-1499, 2006.

57. Stevens TJ, McKenna MJ, Canny BJ, Snow RJ, McConell GK. Effects of sodium bicarbonate on muscle metabolism during intense endurance cycling. Medicine and Science in Sports and Exercise 34: 614-621, 2002.

58. Stone M, Sanborn K, Smith L, O’Bryant H, Hoke T, Utter A, Johnson R, Boros R, Hruby J, Pierce K, Stone M, Garner B. Effects of in-season (5-weeks) creatine and pyruvate supplementation on anaerobic performance and body composition in American football players. International Journal of Sports Nutrition 2: 146-165, 1999.

59. Syrotuik D \& Bell G. Acute creatine monohydrate supplementation: A Descriptive physiological profile of responders vs. non-responders. Journal of Strength and Conditioning Research 18 (3) 610-617, 2004.

60. Tarnopolsky M \& MacLennan D. Creatine monohydrate supplementation enhances highintensity exercise performance in males and females. International Journal of Sport Nutrition and Exercise Metabolism 10: 452-463, 2000. 
61. Tipton K, Jeukendrup A, Hespel P. Nutrition for the sprinter. Journal of Sports Sciences 25(1): 5-15, 2007.

62. Tiryaki G \& Atterbom H. The effects of sodium bicarbonate and sodium citrate on $600 \mathrm{~m}$ running time of trained females. Journal of Sports Medicine and Physical Fitness: 35(5), 194-198, 1995.

63. Tokish J, Kocher M, Hawkins R. Ergogenic aids: a review of basic science, performance, side effects, and status in sports. American Journal of Sports Medicine 32: 1543-1553, 2004.

64. Vandenberghe K, Goris M, Van Hecke P, Van Leemputte M, Vanstapel F, Helpel P. Phosphocreatine resynthesis is no affected by creatine loading. Medicine and Science in Sports and Exercise. 31: 236-242, 1999

65. Van Montfoort MC, Van Dieren L, Hopkins W, Shearman JP. Effects of ingestion of bicarbonate, citrate, lactate, and chloride on sprint running. Medicine and Science in Sports and Exercise. 36(7): 1239-1243, 2004.

66. Webster M, Webster MN, Crawford R, Gladden L. Effect of sodium bicarbonate ingestion on exhaustive resistance exercise performance. Medicine and Science in Sports and Exercise: 25, 960-965, 1993.

67. Wyss M \& Kaddurah-Daouk R. Creatine and creatine metabolism. Physiological Reviews: 80(3), 1107-1213, 2000.

68. Ziegenfuss T, Rogers M, Lowery L, Mullins N, Mendel R, Antonio J, Lemon P. Effect of creatine loading on anaerobic performance and skeletal muscle volume in NCAA division I athletes. Nutrition 18: 397-402, 2002. 


\section{APPENDIX A.}

\section{INFORMED CONSENT TO PARTICIPATE IN RESEARCH STUDYING THE EFFECTS OF A SUPPLEMENTATION ON SPRINT PERFORMANCE}

\section{NATURE AND PURPOSE OF STUDY}

A research project on the effects of a combined supplementation on sprint performance is being conducted by James Barber in the Cal Poly Kinesiology Department.

The purpose of this study is to examine the effects of a two-day combined supplementation of creatine monohydrate and sodium bicarbonate on repeated sprint performance. This research will take place in the Kinesiology Human Performance Laboratory in (43-A first floor) at Cal Poly.

You were invited to participate in this study because you are Cal Poly student and are between 18 and 40 years old, are in good health, and exercise regularly. Approximately 15 participants will be in this study. If you decide to participate, you will be asked to report to the Cal Poly Kinesiology Human Performance Laboratory (43-A first floor) to obtain the supplements for 2 days as well as perform repeated bouts of maximal effort on a cycle ergometer on the $3^{\text {rd }}$ day.

\section{PROCEDURES}

If you decide to participate in this study you will first be asked to complete a health history questionnaire and physical activity readiness questionnaire (PAR-Q) form to help screen for any potential complications that may arise as a result of the exercise tests involved with this study. This will take approximately 10 minutes. Your height and weight will also be recorded at this time. If you have no apparent medical conditions, you will then be asked to perform a maximal graded exercise test to investigate if you are fit enough for the study (maximal oxygen uptake of $>40 \mathrm{ml} / \mathrm{kg} / \mathrm{min}$ ). If you are fit enough to participate in the study, you will be asked to complete three conditions. (See below)

\section{Maximal Exercise Test}

Maximal oxygen consumption will be determined by exercising on a stationary bicycle. You will be wearing a heart rate monitor and breathe into a mouthpiece and tube. A clip will be placed on your nose so that you can only breathe through your mouth. You will begin pedaling at a very easy workload as a warm-up. You will continue to pedal at the same rate while the workload is increased slightly every 2 minutes. It will become more and more difficult to maintain your pedaling rate as the test progresses. The test will be stopped when you no longer wish to continue (test usually lasts a total of 8-15 minutes). This test is physically demanding and you may feel fatigued after the test. The results of this standard test will help us determine whether you are fit enough to participate in the rest of the study.

\section{Supplementation and Exercise Testing}

After the maximal exercise test, you will begin the supplementation procedure. You will complete 3 conditions, followed by a 1-week washout period between each condition: 1) creatine supplementation, 2) combined creatine with sodium bicarbonate supplementation, and 3) placebo 
(sugar). You will be randomly assigned to the order of the supplements and both you and the researcher will be blinded to each supplement (i.e. you will not know which supplement you receive). The dosage for each treatment is as follows: a) Placebo (sugar) 4 X 5 g per day for 2 days combined with $.5 \mathrm{~g} / \mathrm{kg}$ body-weight of sugar which will used as a placebo for creatine monohydrate and sodium bicarbonate (b 4 x $5 \mathrm{~g}$ of creatine along with (.5 g per kilogram body weight of a placebo) for 2 days, and c) 4 X $5 \mathrm{~g}$ of creatine monohydrate combined with sodium bicarbonate (.5 g per kilogram body weight) for 2 days.

\section{Testing Protocol}

In the morning of each condition, you will undergo a repeated sprint assessment by performing 6 repeated 10-second Wingate tests. The repeated sprint test will be administered on a cycle ergometer. The sprint test procedures will consist of: (a) 5-minute warm-up with 3 practice sprints (b) at the end of this warm-up, the test administrator will give a 10-second count down for you to build up to your maximal effort. At the end of this countdown, the 10 -second sprint test will start. The cycle ergometer will magnetically add resistance immediately at the start of the sprint. Immediately following each 10 -second sprint, a 60 -second active rest will be administered. This procedure will be repeated 6 times.

\section{Finger Stick Blood Sample}

You will have a finger stick blood sample after each exercise test to determine blood gases (e.g. pH, lactate, and bicarbonate). The total amount of blood collected will be $<1$ teaspoon for the entire study. Universal precautions, as recommended by the CDC, will be used at all times. All researchers will be trained to perform the finger stick. Note: You have the option of not participating in the blood sample analysis.

\section{RISKS AND DISCOMFORTS}

All possible attempts will be made to minimize the risks involved. Trained individuals will conduct all laboratory procedures with your well being as their first priority. All procedures will be explained and demonstrated until you are comfortable with the proposal study.

\section{Supplementation}

Possible side effects of consuming sodium bicarbonate include feelings of nausea, stomach cramping, vomiting, and diarrhea. Creatine side effects are similar, however, to a lesser degree. These risks are minimized when the dose is consumed over a long period of time, is taken in smaller doses instead of at once, and is consumed with generous amounts of water. You will be consuming four doses of 0.15 grams per kilogram body weight. Negative side effects associated with consuming sodium bicarbonate have only been reported in doses of 0.3 grams per kilogram body weight or larger.

\section{Maximal Exercise Tests}

During any type of exercise, especially strenuous exercise, there are slight health risks, along with the possibility of fatigue and muscle soreness. However any health risks are small in subjects who have no prior history of cardiovascular, respiratory or musculoskeletal disease or injury. Any ordinary fatigue or muscle soreness is temporary and usually lasts 24-96 hours. 
We will follow the American College of Sports Medicine guidelines for exercise testing. The exercise test will be stopped if any of the following conditions happen: onset of chest pain; signs of poor circulation, including pallor (changes in skin color), cyanosis (blue skin), or cold and clammy skin; severe shortness of breath; vertigo or confusion; leg cramps; or intermittent claudicating (blood clotting that can cause intense leg pain). First aid and an automated external defibrillator (AED), along with CPR and AED certified personnel, will be on hand (the AED device is kept with Public Safety) to treat any problems that may arise.

Finger stick blood sample

The total amount of blood taken from you during the entire study is extremely small, only about 1 teaspoon, however there are some minor risks involved. To minimize these risks, only trained individuals using sterile technique at all times will take the blood sample. There may be some pain associated with the stick in a finger. Although rare, there can be local infection if the site is not kept clean following the procedure. Some slight bleeding may also occur during the finger stick. There is the possibility of bruising of the skin in the area around the finger stick that poses no health risk and should subside within a few days.

$\underline{\text { Injuries }}$

If you should experience any injuries or emotional distress, please be aware you may contact the campus Heath Center immediately at (805) 756-1211.

Confidentiality

We are required by law to protect your confidentiality. All records and assessment data from this study will be treated as confidential. Your name and the fact that you are in the study will be kept confidential. Information stored on our computer will be password protected. Information on questionnaires will be identified by participant ID and decoded using a list kept in a locked cabinet. Only research staff will have access to the locked cabinet. All questionnaires completed in this study will be shredded within five years after the study's completion.

\section{BENEFITS OF PARTICIPATION}

Participating in these assessments, you will help us determine whether a supplementation improves performance. You will also gain knowledge about your overall aerobic fitness levels.

\section{ALTERNATIVES TO PARTICIPATION}

The only alternative is to not participate in this study.

\section{COMPENSATION FOR YOUR TIME AND EFFORT}

There is no financial compensation for participating in this study. Your participation is strictly voluntary. You will have no costs by participating in this study.

\section{WITHDRAWAL}


Your participation in this study is strictly voluntary. You have the right to choose not to participate or to withdraw your participation at any point in this study without consequence.

\section{QUESTIONS}

If you have questions regarding this study or would like to be informed of the results when the study is completed, please feel free to contact James Barber, the primary researcher by phone at (559)-999-0953 and/or e-mail jjbarber@calpoly.edu, or faculty advisor, Dr. Todd Hagobian (805) 756-7511. If you have questions or concerns regarding the manner in which the study is conducted, you may contact Dr. Steve Davis, Chair of the Cal Poly Human Subjects Committee, at 756-2754, sdavis@calpoly.edu, or Dr. Susan Opava, Dean of Research and Graduate Programs, at 756-1508, sopava@calpoly.edu.

If you agree to voluntarily participate in this research project as described, please indicate your agreement by completing and returning the attached questionnaires. Please retain this consent cover form for your reference, and thank you for your participation in this research.

\section{SIGNATURE}

You will be given a copy of this consent form to keep. By signing this consent form, you are not waiving any of your legal rights, claims, or remedies. If you have questions about participation contact James Barber at (559) 999-0953.

I have read this consent form. I agree to take part in the research. I have had an opportunity to ask questions and all of my questions have been answered to my satisfaction. By signing this consent form, I willingly agree to participate in this study.

Name of Subject (type or print)

\section{Signature of Subject}

\section{Date}

I have explained the research to the subject and answered all of his/her questions. I believe that she understands the information described in this consent form and freely consents to participate. I have fully explained to the above volunteer the nature and purpose, procedures, and possible risks of the research study.

Name of Investigator/Research Team Member (type or print)

(Signature of Investigator/Research Team Member) 


\title{
APPENDIX B.
}

\author{
Health \& Fitness History
}

Name: E-mail: Date of Birth:

Phone: Home Work: Height: Weight:

1. Have you ever been diagnosed as having any of the following and if yes, how are you currently treating the condition?

Y N High Blood Pressure

Y N High Cholesterol or High Triglycerides

Y N Diabetes

Y N Hypoglycemia (low blood sugar)

Y N Asthma

2. Does anyone in your family (immediate family including your grandparents) have a history of cardiovascular disease? (heart attacks, strokes, etc.) Please explain:

3. Does anyone in your family (immediate family including your grandparents) have a history of type 2 diabetes? Please explain:

4. Have you ever had a glucose tolerance test? $\mathrm{Y} \quad \mathrm{N}$

If yes, what were the results?

5. Have you ever had a fasting blood sugar test? $\mathrm{Y} \quad \mathrm{N}$ If yes, what were the results?

6. For women:

- Did you have gestational diabetes during any pregnancy?

- Were you ever tested for gestational diabetes?

- How much did your children weigh at birth?

- For women: are you on hormonal birth control (pill, patch, etc) or estrogen replacement? Describe in detail: 
- What was the date when your last period started? Is your cycle regular?

7. Do you have any neurological problems including fainting, dizziness, headaches or seizures?

8. Do you have any orthopedic or other health problems that may affect your ability to perform exercise? If yes, please explain.

9. Are you currently taking any medications, including over-the-counter drugs such as aspirin, Tylenol or Ibuprofen? Please list:

10. Do you smoke or use smokeless tobacco?

11. Do you drink coffee or other caffeinated beverages? $\mathrm{Y} N$ What kind, how much and how often?

12. Please list all vitamins, minerals and herbs and other nutritional supplements you're taking:

13 Do you have any food allergies or intolerances? Please describe:

14. How would you describe the type of diet you currently eat? Have you recently been on any special diets? What kinds of diets have you used to lose weight or lower cholesterol? Please list and describe:

15. What changes have you made in your diet in the last 6 months?

16. Do you exercise regularly? What kinds of exercise?

How often?

Please describe how much walking you do on a daily basis: 
17. How does your current exercise and physical activity compare to 6 months ago? 1 year ago?

18. Do you have a history of Orthorexia (using excessive exercise or diet to stay thin or fit) ? Y $\mathrm{N}$

If so, when were you diagnosed?

19. Do you have any history of Bulimia (in which exercise is used as a purging tool)?

20. How much did you weigh at birth (if known)?

21. Have you had a physical exam in the past two years? $\mathrm{Y} N$ Please describe your assessment of your overall health:

22. Have you ever supplemented on creatine or sodium bicarbonate before? Y N If so, how long ago did you last ingest either?

Participant's Signature:

Date: 


\section{APPENDIX C.}

Subject Name:

Fatigue and GI Distress Questionnaire

Date:

Fatigue Rating 1-10 (From Multidimensional Fatigue Questionnaire)

\begin{tabular}{|l|l|l|l|l|l|l|}
\hline & Sprint \#1 & Sprint \#2 & Sprint \#3 & Sprint \#4 & Sprint \#5 & Sprint \#6 \\
\hline $\begin{array}{l}\text { Fatigue } \\
\text { Rating }\end{array}$ & & & & & & \\
\hline
\end{tabular}

Scale: $(1=$ none at all to $10=$ a great deal

\section{$\underline{\text { GI Distress Assessment }}$}

Question 1: To what degree are you feeling upper abdominal pains? (e.g. bloating, cramps vomiting, or nausea?

Question 2: To what degree are you feeling lower abdominal pains? (e.g. intestinal cramps, flatulence, loose stool, or diarrhea)

Question 3: To what degree are you feeling systemic problems? (e.g. dizziness, headache, muscle cramps, urge to urinate)

\begin{tabular}{|l|l|l|l|l|l|l|}
\hline & Sprint \#1 & Sprint \#2 & Sprint \#3 & Sprint \#4 & Sprint \#5 & Sprint \#6 \\
\hline $\begin{array}{l}\text { Upper } \\
\text { abdominal } \\
\text { pain }\end{array}$ & & & & & & \\
\hline $\begin{array}{l}\text { Lower } \\
\text { Abdominal }\end{array}$ & & & & & & \\
Pain & & & & & & \\
\hline $\begin{array}{l}\text { Systemic } \\
\text { Problems }\end{array}$ & & & & & & \\
\hline
\end{tabular}

Scale: (1: no problem at all-10: the worst it has ever been)

NOTES: 


\section{APPENDIX D.}

\section{Supplement Protocol}

\section{Supplement and Dietary Recall Instructions}

Please mix the drinks WITH WATER ONLY! Drink the entire dose within the specified time below:

\begin{tabular}{|l|l|l|}
\hline Bottle $\#$ & Time & Actual time ingested \\
\hline 1 & $9 \mathrm{am}$ & \\
\hline 2 & $12 \mathrm{pm}$ & \\
\hline 3 & $6 \mathrm{pm}$ & \\
\hline 4 & $10 \mathrm{pm}$ & \\
\hline & & \\
\hline
\end{tabular}

Drink the bottle at least 20 minutes before you eat any food. Drink with plenty of water. On the day of the test, please do not ingest any food or drinks with calories before the exercise test. You may bring water to your exercise test.

\section{Dietary Recall}

Please complete a dietary intake log (attached sheet) for both days of the supplementation period.

\section{PLEASE NOTE: Do NOT ingest caffeine or alcohol during the supplementation period. These substances interact with the supplementation.}

\section{Hydration}

Please consume at least 2 liters of water on each day of the supplementation period.

Actual Amount

$\underline{\text { Exercise }}$

Please refrain from exercise 24 hours before the testing protocol.

\section{Agreement}

To the best of my knowledge, I have complied with these instructions for supplementation, dietary intake, hydration, and exercise procedures for this research project.

Sign

Date 
